\title{
Distinct mechanisms govern populations of myeloid-derived suppressor cells in chronic viral infection and cancer
}

\author{
Evgenii N. Tcyganov, ${ }^{1}$ Shino Hanabuchi, ${ }^{2}$ Ayumi Hashimoto, ${ }^{1,2}$ David Campbell, ${ }^{2}$ Gozde Kar, ${ }^{3}$ Timothy W.F. Slidel, ${ }^{3}$ \\ Corinne Cayatte, ${ }^{2}$ Aimee Landry, ${ }^{2}$ Fernanda Pilataxi, ${ }^{2}$ Susana Hayes, ${ }^{2}$ Brian Dougherty, ${ }^{2}$ Kristin C. Hicks, ${ }^{2}$ Kathy Mulgrew, ${ }^{2}$ \\ Chih-Hang Anthony Tang, ${ }^{1}$ Chih-Chi Andrew Hu, ${ }^{1}$ Wei Guo, ${ }^{4}$ Sergei Grivennikov, ${ }^{4}$ Mohammed-Alkhatim A. Ali, ${ }^{5}$ \\ Jean-Christophe Beltra, ${ }^{5,6,7}$ E. John Wherry, ${ }^{5,6,7}$ Yulia Nefedova, ${ }^{1}$ and Dmitry I. Gabrilovich ${ }^{2}$ \\ IImmunology, Microenvironment, and Metastasis Program, The Wistar Institute, Philadelphia, Pennsylvania, USA. ${ }^{2}$ AstraZeneca, Gaithersburg, Maryland, USA. ${ }^{3}$ AstraZeneca, Translational Medicine, Research \\ and Early Development, Oncology Research \& Development, Cambridge, United Kingdom. ${ }^{4}$ Cancer Prevention and Control Program, Fox Chase Cancer Center, Philadelphia, Pennsylvania, USA. ${ }^{5}$ Department \\ of Systems Pharmacology and Translational Therapeutics and ${ }^{6}$ Institute for Immunology, Perelman School of Medicine, University of Pennsylvania, Philadelphia, Pennsylvania, USA. ${ }^{7}$ Parker Institute for \\ Cancer Immunotherapy at University of Pennsylvania, Philadelphia, Pennsylvania, USA
}

\begin{abstract}
Myeloid-derived suppressor cells (MDSCs) are major negative regulators of immune responses in cancer and chronic infections. It remains unclear if regulation of MDSC activity in different conditions is controlled by similar mechanisms. We compared MDSCs in mice with cancer and lymphocytic choriomeningitis virus (LCMV) infection. Chronic LCMV infection caused the development of monocytic MDSCs (M-MDSCs) but did not induce polymorphonuclear MDSCs (PMN-MDSCs). In contrast, both MDSC populations were present in cancer models. An acquisition of immune-suppressive activity by PMNMDSCs in cancer was controlled by IRE1 $\alpha$ and ATF6 pathways of the endoplasmic reticulum (ER) stress response. Abrogation of PMN-MDSC activity by blockade of the ER stress response resulted in an increase in tumor-specific immune response and reduced tumor progression. In contrast, the ER stress response was dispensable for suppressive activity of M-MDSCs in cancer and LCMV infection. Acquisition of immune-suppressive activity by M-MDSCs in spleens was mediated by IFN- $\gamma$ signaling. However, it was dispensable for suppressive activity of M-MDSCs in tumor tissues. Suppressive activity of M-MDSCs in tumors was retained due to the effect of IL- 6 present at high concentrations in the tumor site. These results demonstrate disease- and population-specific mechanisms of MDSC accumulation and the need for targeting different pathways to achieve inactivation of these cells.
\end{abstract}

\section{Introduction}

Myeloid-derived suppressor cells (MDSCs) with potent immune-suppressive activity are widely implicated in negative regulation of immune responses in many pathological conditions including cancer, chronic inflammation, infections, autoimmune diseases, and others (1). Accumulation of MDSCs was directly linked with a negative clinical outcome in cancer, as well as poor clearance of chronic infections and sepsis (2-5). Two large groups of MDSCs are currently recognized: pathologically activated polymorphonuclear neutrophils (PMN-MDSCs) and pathologically activated monocytes (M-MDSCs). Although MDSCs and their classical counterparts, neutrophils (PMNs) and monocytes (MONs), share many phenotypic and morphological characteristics, they have distinct transcriptomic and proteomic profiles, metabolism, biochemical features, and functions (1, 6-10). It

Conflict of interest: SH, AH, DC, GK, TWFS, CC, AL, FP, SH, BD, KCH, KM, and DIC are employees of AstraZeneca.

Copyright: ( 2021, American Society for Clinical Investigation.

Submitted: November 11, 2020; Accepted: July 1, 2021; Published: August 16, 2021

Reference information: J Clin Invest. 2021;131(16):e145971

https://doi.org/10.1172/JCl145971. is established that expansion of immature myeloid cells is governed by increased production of different growth factors such as GM-CSF or M-CSF. However, the factors responsible for acquisition of immune-suppressive activity by these cells remain largely elusive. An understanding of these mechanisms would be important to develop selective targeting of MDSCs. The major characteristics of MDSCs in chronic infections and cancer are believed to be the same (1). However, whether mechanisms regulating MDSC accumulation are shared between chronic infections and cancer remains unclear. By using different experimental models of viral infection and cancer we sought to identify possible common mechanisms responsible for acquisition of immune-suppressive activity by MDSCs.

Previously, we and others have demonstrated that MDSCs, in contrast to MONs or PMNs, displayed signs of endoplasmic reticulum (ER) stress $(7,11-13)$. The ER stress response, also known as the unfolded protein response (UPR), is a common mechanism developed to protect cells from various conditions, including starvation, viral infections, oxidative stress, and so on. Synthesis and folding of integral transmembrane and secretory proteins take place in the ER. Only correctly folded proteins are exported via the trans-Golgi network to the plasma membrane or outside of 
the cells (14). In eukaryotes, the UPR consists of 3 major signaling cascades (15). Upon the detection of accumulating misfolded proteins, protein kinase RNA-like (PKR-like) ER kinase (PERK) phosphorylates eukaryotic protein synthesis initiation factor 2 (eIF2a), which inhibits initiation of mRNA translation and halts the flux of newly synthetized proteins into the ER. eIF2a activates the expression of the transcription factor ATF 4 and its downstream targets, including the proapoptotic transcription factor C/EBP homologous protein (CHOP) as well as the growth arrest and DNA damage-inducible protein 34 (GADD34), a phosphatase 1 cofactor that functions as a negative-feedback regulator of eIF2a phosphorylation. Inositol-requiring enzyme 1 (IRE1) cleaves the mRNA encoding for the transcription factor X-box-binding protein 1 (Xbp1) (16). Cleaved XBP1 mRNA is then ligated by a tRNA ligase, RtcB, turning into spliced version (sXBP1) and translated to produce sXbp1 protein. $\mathrm{SXBP} 1$ is a highly active transcription factor and one of the key regulators of ER folding capacity, lipid biosynthesis, and ER-associated degradation (17). IRE1 activity also includes degradation of a specific set of mRNAs via the process of regulated IRE1-dependent decay (RIDD). Activating transcription factor 6 (ATF6) is transported to the Golgi apparatus and processed into an active transcription factor. After nuclear translocation, ATF6 induces the transcription of ER chaperone genes, such as $\mathrm{BiP}$ and several major targets of the mammalian UPR, including Xbp1.

In this study, we focused on the ER stress response possibly being a common pathway responsible for acquisition of MDSC activity in chronic viral infection and cancer. We expected to identify the role of specific sensors of ER stress in the regulation of MDSCs. Surprisingly, we determined that acquisition of PMN-MDSC and M-MDSC suppressive activity in viral infection and cancer was governed by different basic mechanisms. PMN-MDSC activity in cancer was regulated by the IRE1 $\alpha$ and ATF6 pathways of the ER stress response, whereas both pathways were dispensable for functional activity of M-MDSCs. Instead, in both viral infection and cancer, M-MDSC function was dependent on IFN- $\gamma$ signaling and independent of ER stress. Specific targeting of these mechanisms had different functional consequences for generation of antiviral and antitumor immune responses.

\section{Results}

Accumulation of MDSC populations in viral infection and cancer. To assess the effect of viral infection on MDSCs, we used 2 strains of LCMV: Armstrong (LCMV-Arm), which causes acute infection and rapid clearance of virus, and C13 (LCMV-C13), which is not rapidly cleared by the host immune system and causes chronic infection (18). On day 7 after infection we observed similar

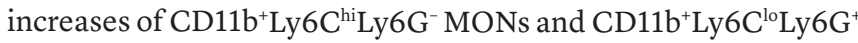
PMNs for both acute and chronic infection models. However, by day 14 after infection, the myeloid compartment (most prominently MONs) started to come back to baseline levels in the acute infection model, whereas it maintained significantly elevated levels in chronically infected mice and returned to the values of naive mice by day 30 after infection (Supplemental Figure 1; supplemental material available online with this article; https://doi. org/10.1172/JCI145971DS1). Suppressive activity of these cells was assessed in antigen-specific response of OT1 T cells. Spleen MONs from mice infected with LCMV-Arm had no suppressive activity at any time after infection. In contrast, in LCMV-C13infected mice, suppressive activity was detected on day 7 after infection. It became more prominent by day 14 and, although decreased, was still detectable on day 30 after infection (Figure 1A). In contrast, no suppressive activity was detected in PMNs in either acute or chronic LCMV infection (Figure 1B). Thus, consistent with a previous report (19), infection with LCMV-C13 but not with LCMV-Arm caused accumulation of immune-suppressive M-MDSCs. However, we did not find immune-suppressive PMN-MDSCs in these mice. Remarkably, suppressive activity did not match expansion of myeloid cells. PMNs were expanded much stronger than MONs but had no suppressive activity. The population of M-MDSCs returned to control values by day 30, but suppressive activity in these cells was still detected.

To assess function of MDSCs in cancer, we used 2 tumor models: Lewis Lung Cancer (LLC) and EL4 lymphoma. Myeloid cells were evaluated 3 weeks after tumor inoculation. Both tumor models caused accumulation of MONs and PMNs (Supplemental Figure 2) in spleens and tumors with strong immune-suppressive activity, indicative of M-MDSCs and PMN-MDSCs, respectively (Figure 1, C and D).

Since suppressive M-MDSCs were found in the models of viral infection and cancer, we asked if these cells were functionally different. M-MDSCs are characterized by a well-defined set of genes implicated in their immune-suppressive activity (6). We found that M-MDSCs from mice infected with LCMV-C13 and M-MDSC from tumors of LLC tumor-bearing (TB) mice had similar substantial upregulation of the expression of Nos2, Arg1, and Il-1O (Figure 2). In addition, tumor infiltrating M-MDSCs upregulated Ptges expression. In splenic M-MDSCs we observed the upregulation (about 8-fold increase) of Nos2, but not of the other genes (Figure 2). To better understand the difference between M-MDSCs from LCMV-C13infected and LLC TB mice, we performed whole transcriptome RNA sequencing (RNAseq). We compared changes in gene expression between spleen MONs from control mice and spleen M-MDSCs from LCMV-C13-infected and LLC TB mice isolated at the peak of their suppressive activity (day 14 for LCMV-C13-infected mice and day 21 for TB mice). There was a strong correlation in differentially expressed genes between M-MDSCs from LCMV-C13-infected and LLC TB mice (Figure 3A). However, the number of genes changed differently in M-MDSCs from LCMV-C13 and LLC TB mice (Figure 3B). Most of the pathways changed similarly in M-MDSCs from LCMV-C13-infected and LLC TB mice. Notably, enrichment analysis showed similar upregulation of E2F target genes in M-MDSCs from LCMV-C13-infected and LLC TB mice (Figure 3C). E2F is an important component of ER stress pathways. M-MDSCs from LCMV-C13-infected mice demonstrated upregulation of genes associated with interferon gamma and interferon alpha responses. However, this effect was absent in M-MDSCs from LLC TB mice (Figure 3C). Thus, although M-MDSCs in LCMV-C13-infected and LLC TB mice share major characteristics of immune-suppressive activity, there were some differences in gene expression profile between these cells.

The ER stress response regulates the functional activity of PMN-MDSCs. Previously, we and others have implicated the ER stress response in acquisition of suppressive activity by MDSCs $(7,11,12)$. However, the direct contribution of each specific ER 
A

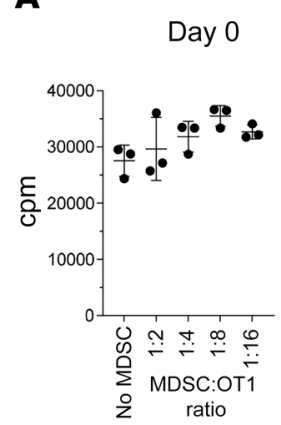

B

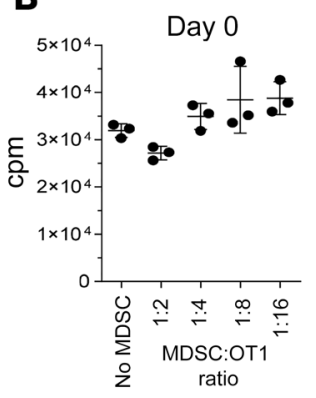

C
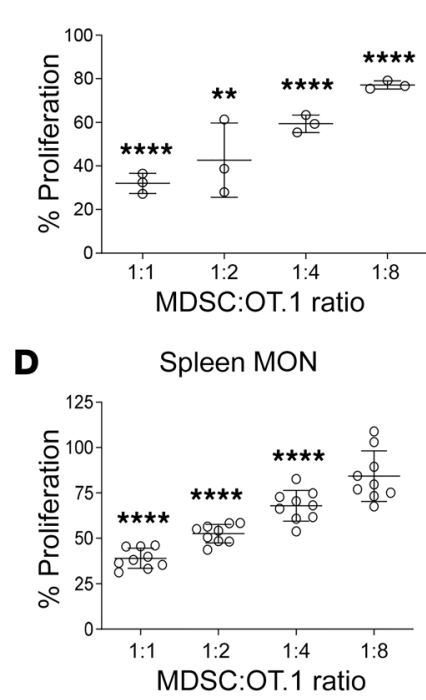

Day 7

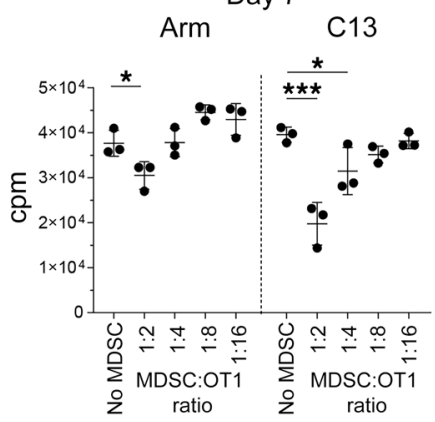

Day 7

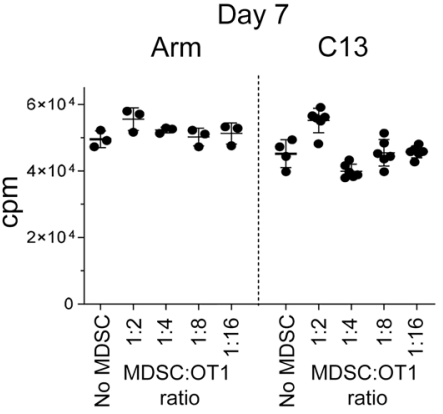

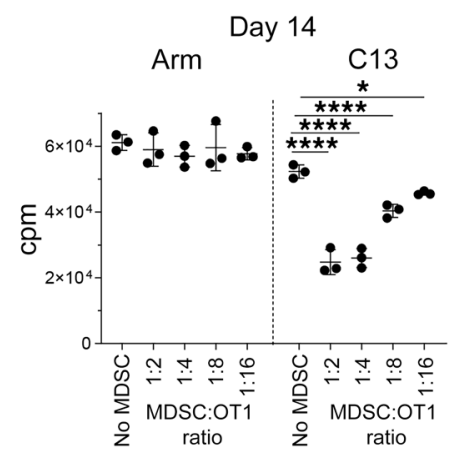

Day 14

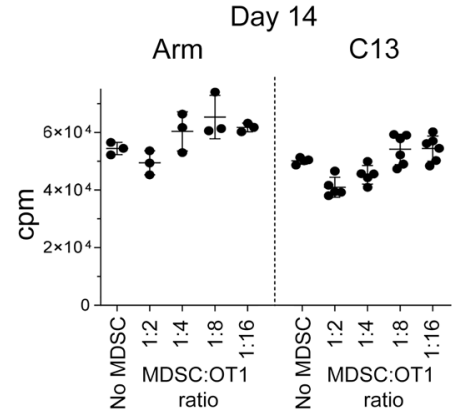

Day 30

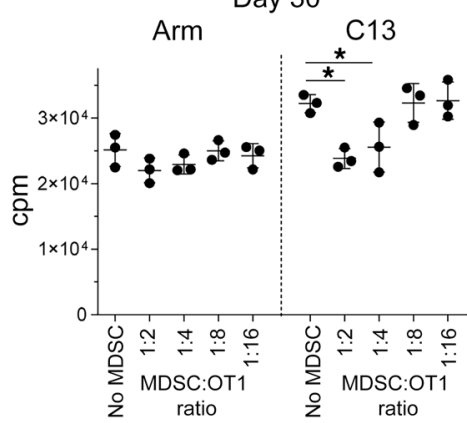

Day 30

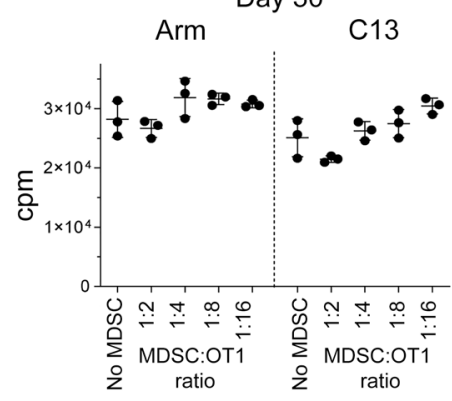

Spleen PMN

Tumor MON

Tumor PMN
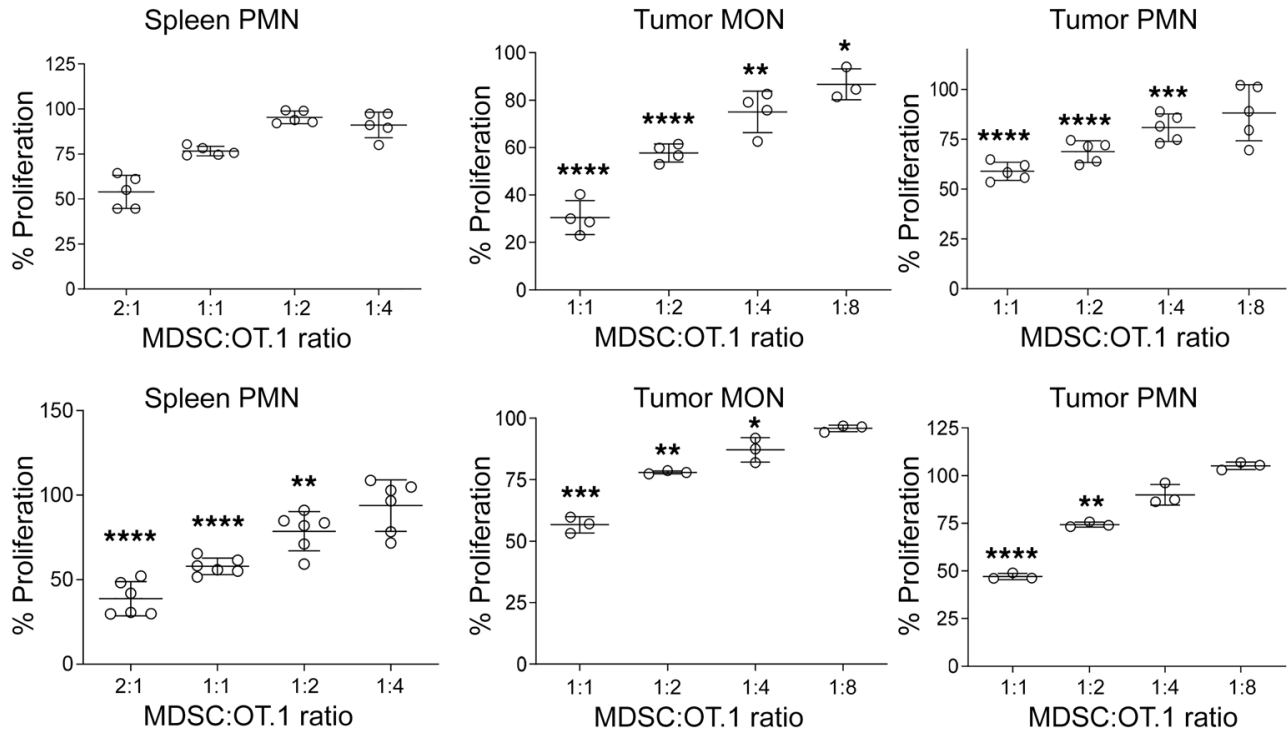

Figure 1. Viral infection and tumors induce different subsets of MDSCs. (A and B) Mice were infected with LCMV-Arm or LCMV-C13 viruses. Monocytes (A) and neutrophils (B) were sorted from pooled 3 to 4 murine spleens at specified time-points and their suppressive activity was measured in co-cultures with activated OT.1 splenocytes. The representative experiment out of 3 independent experiments with similar results is shown. (C and D) Similar experiments as in A and $\mathbf{B}$ were conducted using LLC (C) and EL4 (D) tumor-bearing mice. Monocytes and neutrophils were sorted from spleens and tumors and their suppressive activity was measured in co-cultures with activated OT.1 splenocytes. The representative experiment of 3 independent experiments with similar results is shown. Proliferation of T cells in the absence of MDSCs in each experiment was set as $100 \%$. ${ }^{*} P<0.05,{ }^{* *} P<0.01 ;{ }^{* * *} P<0.001,{ }^{* * * *} P<$ 0.0001 from control in ANOVA test with correction for multiple comparisons.

stress response pathway to MDSC function remained unclear. We assessed the expression of genes associated with all 3 major pathways of the ER stress response: PERK, IRE1 $\alpha$, and ATF6. Myeloid cells isolated from either LCMV-Arm- or LCMV-C13-infected mice didn't show prolonged upregulation of transcripts or proteins of ER stress response pathways that would correlate with suppressive activity of M-MDSCs (Supplemental Figure 3). In contrast, tumor-associated M-MDSCs had significant upregulation of the expression of Chop (part of PERK pathway) and Erp72 (down- stream from IRE1 $\alpha$ and ATF6 pathways; Figure 4A). In PMNMDSCs from spleens of LLC TB mice, upregulation of Xbp1s (IRE1 $\alpha$ pathway) was observed. That increase was much more prominent in tumors. In addition to the IRE1 $\alpha$ pathway, upregulation of components of the PERK and ATF6 pathways was also observed in tumor-infiltrating PMN-MDSCs (Figure 4B).

To directly address the contribution of the ER stress response to MDSC function, we used mice with deletion of each ER stress response pathway: Ire $1 \alpha^{\mathrm{t} / \mathrm{f}} L y s M$-Cre mice with targeted deletion 

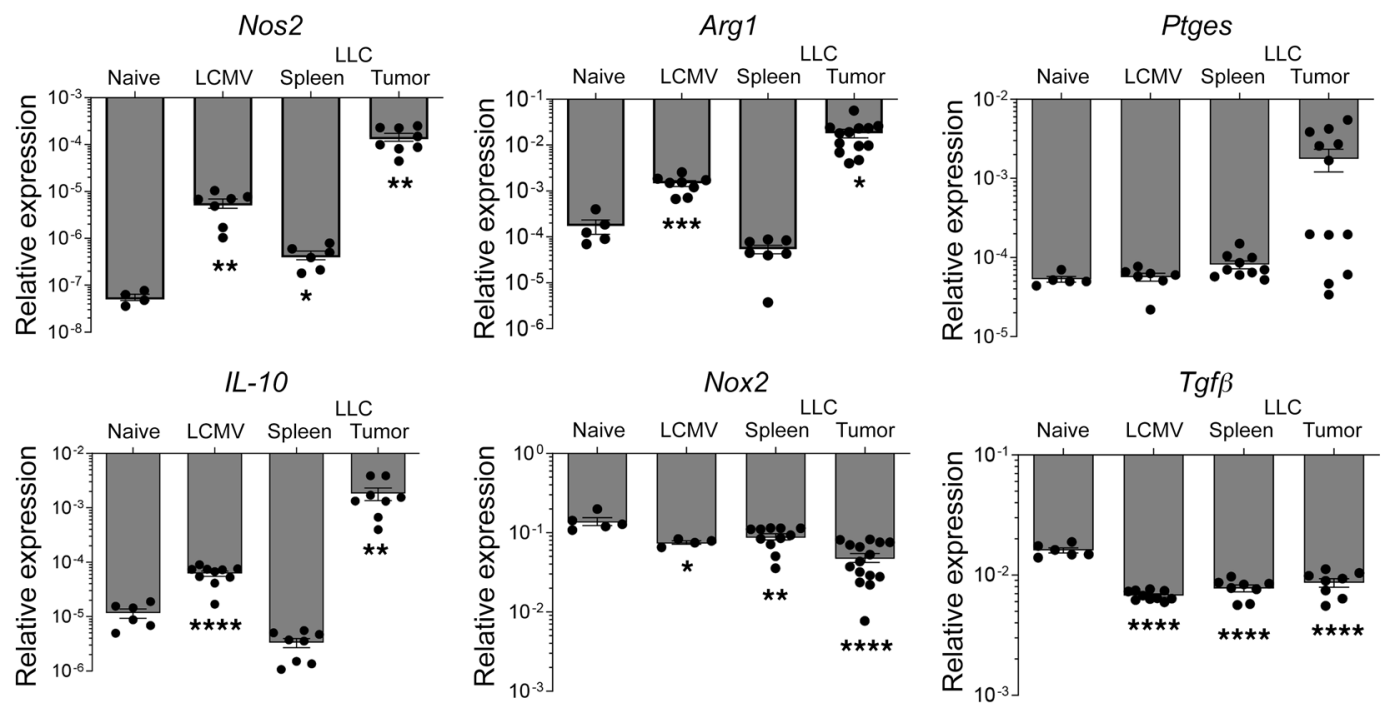

Figure 2. Expression of the genes implicated in M-MDSC suppressive activity. Monocytic cells were sorted from spleens of naive, C13-LCMV-infected (day 14) mice and spleens and tumors of LLC TB mice. The expression of the indicated genes adjusted to $\beta$-actin expression was measured by qRT-PCR $(n=4-13)$. Individual values are shown on graphs. ${ }^{*} P<0.05,{ }^{* *} P<0.01 ;{ }^{* *} P<0.001$, ${ }^{* * *} P<0.0001$ from control using ANOVA test with correction for multiple variables.

of IRE1 $\alpha$ in myeloid cells (IRE1 $\alpha^{\Delta \text { Myel })}$ ) Atf $6^{\mathrm{n} / \mathrm{l}} L y s M$-Cre deletion of ATF6 in myeloid cells (ATF6 $\left.6^{\triangle \mathrm{Myel}}\right)$, and total CHOP-KO. In IRE1 $\alpha^{\Delta \text { Myel }}$ and ATF $6^{\Delta \text { Myel }}$ mice, the expression of targeted genes in PMNs and MONs was decreased by 50\% to $70 \%$ (Supplemental Figure 4A). We also confirmed lack of the expression of Chop in CHOP-KO mice after stimulation of splenocytes with thapsigargin (ER stress inducer; Supplemental Figure 4A). Consistent with the absence of upregulation of the ER stress pathways in M-MDSCs from LCMV-C13 mice, deletion of CHOP, IRE1 $\alpha$, or ATF6 did not cancel potent suppressive activity of M-MDSCs in mice infected with LCMV-C13. PMNs in WT LCMV-C13-infected mice were not suppressive and this was not changed in KO mice (Supplemental Figure 4B).

Similar experiments were performed in LLC TB mice. Deleting any of the 3 targeted genes did not affect tumor growth (Figure $5 \mathrm{~A}$ ) and did not affect suppressive activity of M-MDSCs (Figure 5, B-D). However, deletion of IRE1 $\alpha$ completely abrogated suppressive activity of PMN-MDSCs in spleens and tumors (Figure 5B), and deletion of ATF6 abrogated suppressive activity of PMN-MDSCs in tumors, but not in spleens (Figure 5C), whereas deletion of $\mathrm{CHOP}$ had no effect on suppressive activity of PMN-MDSCs (Figure 5D).

In contrast to LLC, MC38 is characterized by overexpression of p53 and considered to be a moderately immunogenic tumor (no spontaneous rejection, but highly susceptible to immune therapy with check-point inhibitors). We observed a significant delay of tumor growth in IRE1 $\alpha^{\Delta \mathrm{Myel}}$ and ATF $6^{\Delta \mathrm{Myel}}$ mice, but not in CHOPKO mice (Figure 6A). The observed antitumor effect was $\mathrm{CD} 8^{+} \mathrm{T}$ cell-mediated, since the depletion of $\mathrm{CD} 8^{+} \mathrm{T}$ cells abrogated this effect (Figure 6B). MC38 tumors express the p53-derived peptide KYMCNSSCM. We observed a significantly higher spontaneous peptide-specific $\mathrm{CD} 8^{+} \mathrm{T}$ cell response in MC38 TB IRE1 $\alpha^{\triangle \mathrm{Myel}}$ and ATF6 ${ }^{\Delta \text { Myel }}$ mice than in control mice. No such effect was seen in CHOP-KO mice (Figure 6C), indicating that IRE1 $\alpha$ or ATF6 deletion in MDSCs substantially enhanced tumor-specific immune response. IRE1 $\alpha$ deletion resulted in a substantial decrease in the expression of Nos2 in tumor PMN-MDSCs, whereas no effect on the expression of Nox2 or Arg1 was observed. (Figure 6D). In spleen PMN-MDSCs, IRE1 $\alpha$ deletion inhibited the expression of Arg1 (Figure 6E). IRE1 $\alpha^{\triangle \mathrm{Myel}}$ tumor-infiltrating PMN-MDSCs produced less immune-suppressive $\mathrm{PGE}_{2}$ than PMN-MDSCs in control Cre- TB mice. A similar effect was observed in PMN-MDSCs from $\mathrm{ATF}^{\triangle \mathrm{Myel}} \mathrm{TB}$ mice (Figure $6 \mathrm{~F}$ ). These results indicate that tumor PMN-MDSC activity is mainly controlled by activation of the IRE1 $\alpha$ and ATF6 pathways of the ER stress response involving upregulation of Nos2, Arg1, and $\mathrm{PGE}_{2}$ production. However, the ER stress response was dispensable for M-MDSC activity in both tumor and viral infection models.

Mechanism of M-MDSC suppressive activity in LCMV infection and cancer. In contrast to PMN-MDSCs, IRE1 $\alpha$ and ATF6 did not control suppressive activity of M-MDSCs in TB mice (Figure 5). Similarly, M-MDSC suppressive activity was not abrogated by targeting the ER stress response in LCMV-C13-infected mice (Supplemental Figure 4B). We asked: What could be the mechanism of M-MDSC induction? Analysis of serum cytokines performed in LCMV-C13 and LCMV-Arm mice demonstrated a substantial increase of IFN- $\gamma$ in the plasma of LCMV-C13 mice at the time of M-MDSC suppressive activity (Figure 7A). This was consistent with upregulation of the interferon gamma pathway observed in M-MDSCs from LCMV-C13-infected mice by RNAseq (Figure 3C). To test the role of IFN- $\gamma$ directly, we used Ifngr $2^{f / / 1} L y s M-\mathrm{Cre}^{+}$ mice with targeted deletion of IFN- $\gamma$ receptor in myeloid cells (IFN- $\gamma$ R2 ${ }^{\Delta \text { Myel }} ;$ Supplemental Figure 5). Deletion of IFN- $\gamma$ R2 completely abrogated suppressive activity of M-MDSCs in LCMV-C13 mice (Figure 7B). We assessed the effect of IFN- $\gamma$ R2 deletion on generation of LCMV-specific immune response in mice 2 weeks after infection with LCMV-C13. CD8 ${ }^{+} \mathrm{T}$ cell responses specific for LCMV-derived gp33-41 peptide were measured by intracellular staining in $\mathrm{CD} 8^{+} \mathrm{T}$ cells (Figure $7 \mathrm{C}$ ) and IFN- $\gamma$ ELISPOT (Figure 

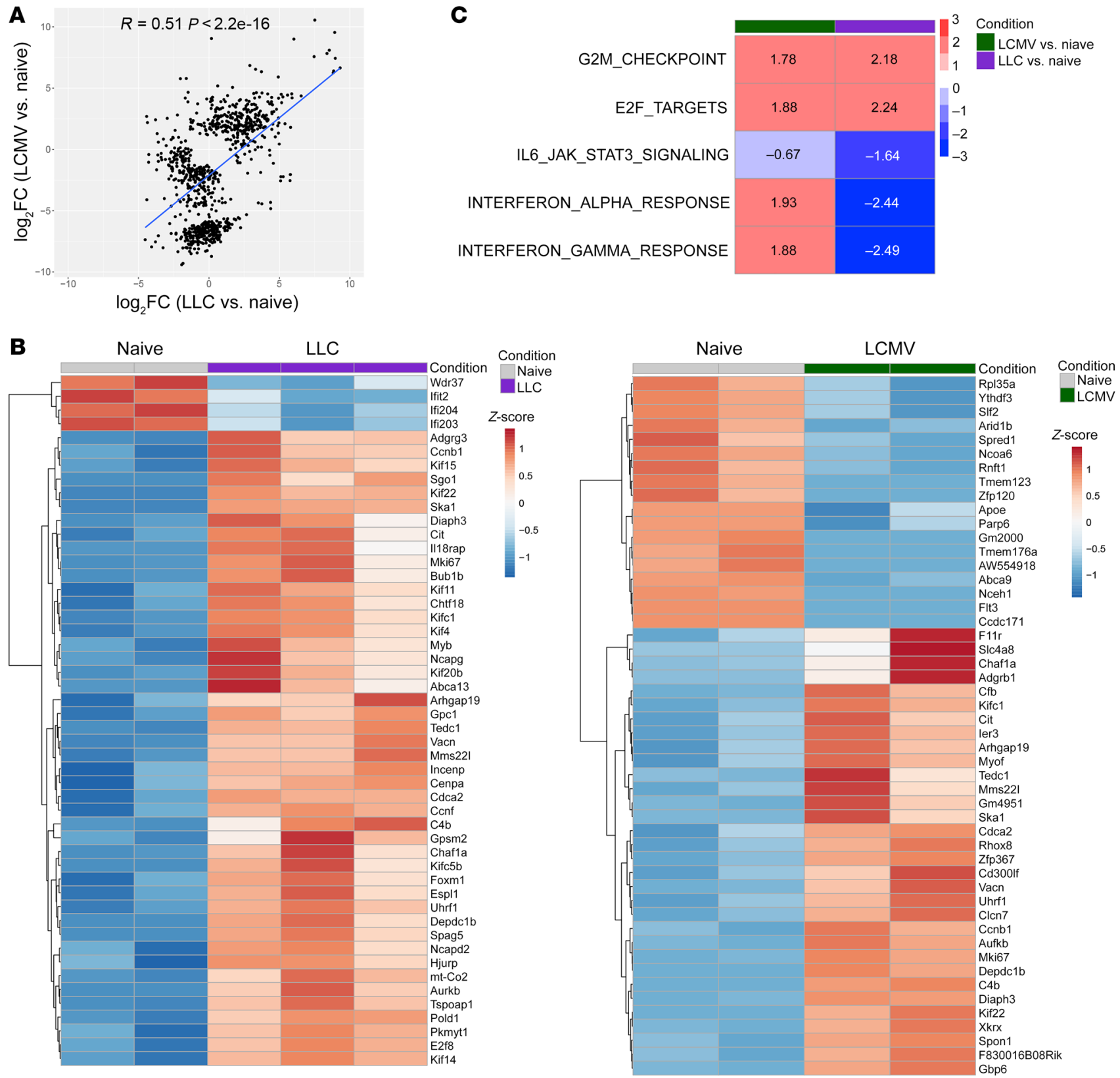

Figure 3. Gene expression profile of M-MDSCs. (A) Correlation of $\log _{2} F C$ of differentially expressed genes in spleen M-MDSCs from LLC TB mice versus MONs from naive mice and M-MDSCs from LCMV-C13-infected mice versus MONs from naive mice. (B) Heatmap showing top 50 differentially expressed genes for M-MDSCs from LLC TB mice versus MONs from naive mice and M-MDSCs from LCMV-13-infected mice versus MONs from naive mice. (C) Gene set enrichment analysis represents NES (normalized enrichment score) as calculated by fCSEA.

7D). In both assays, we observed substantially higher responses in IFN- $\gamma \mathrm{R}^{2 \mathrm{Myyl}}$ than in Ifngr $2^{\mathrm{Al} / \mathrm{l}}$ control mice (Figure 7, C and D). Deletion of IFN- $\gamma$ R2 in M-MDSCs dramatically reduced the expression of Nos2 without affecting Arg1, Nox2, or Ptges (Figure 7E). To confirm a functional role of NO production by M-MDSCs, we used the selective iNOS (NOS2) inhibitor L-NMMA. Inhibition of NOS2 abrogated suppressive activity of M-MDSCs (Figure 7F). Thus, M-MDSC activity in chronic LCMV infection was regulated by IFN- $\gamma$ via upregulation of Nos2.

Similar experiments were performed in LLC and MC38 TB mice. IFN- $\gamma$ was undetectable in spleens of tumor-free mice. It was high- er (around $300 \mathrm{pg} / \mathrm{g}$ ) in spleens of TB mice. In tumors, IFN- $\gamma$ was much higher (around 1200 pg/g; Figure 8A). Growth of LLC was not affected in IFN- $\gamma \mathrm{R}^{\mathrm{MMyel}}$ mice (Figure 8B). Although there was some trend in delay of growth of MC38 tumor in IFN- $\gamma \mathrm{R} 2^{\mathrm{\Delta Myel}}$ mice, it also did not reach significance (Figure 8C). Deletion of IFN- $\gamma$ R2 did not affect suppressive activity of PMN-MDSCs in spleens or tumors. IFN$\gamma \mathrm{R} 2$ deletion abrogated suppressive activity of M-MDSCs in spleens, but not in tumors (Figure 8D). Deletion of IFN- $\gamma$ R2 caused decrease of Nos 2 expression. However, it was observed only in spleen but not in tumor M-MDSCs (Figure 8E). Apparently, the interferon gamma pathway contributed to suppressive activity of splenic M-MDSCs. 
A

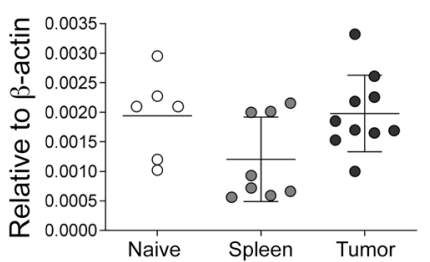

Sec61a

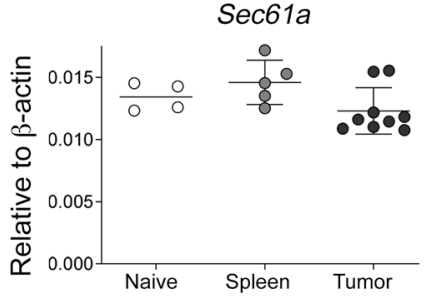

B

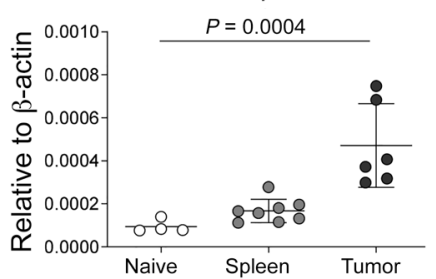

Sec61a

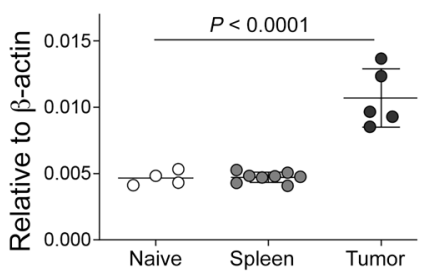

Atf6

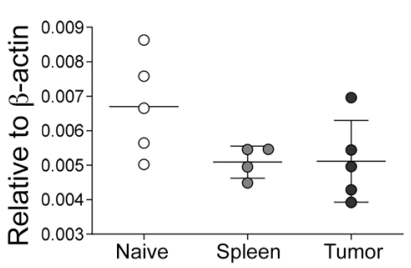

Erdj4

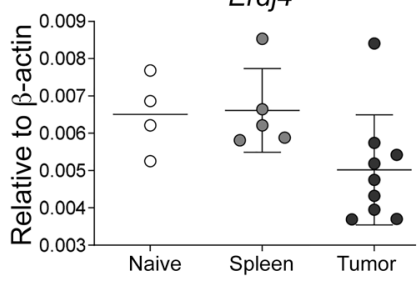

Atf6

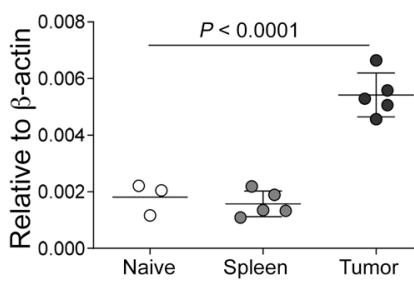

Erdj4

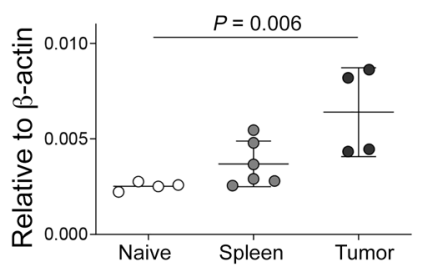

Erp72

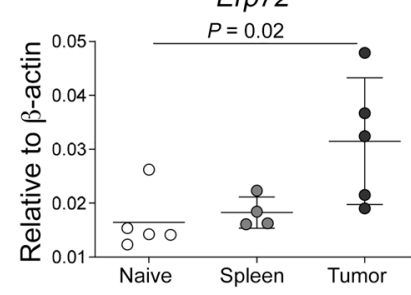

CHOP

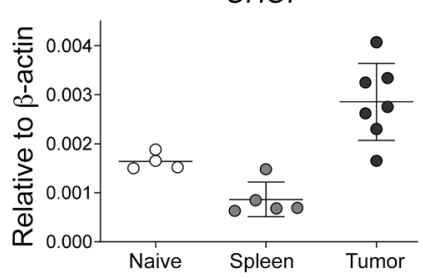

Erp72

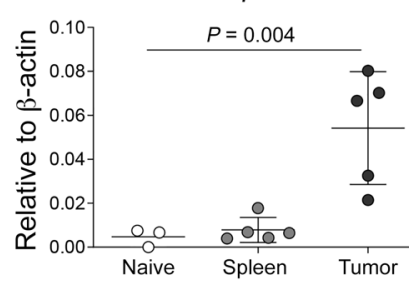

Chop

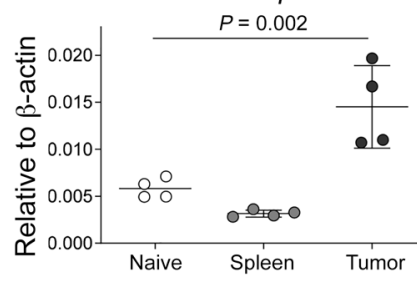

Dnajb11

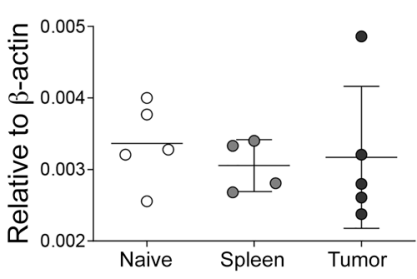

atf4

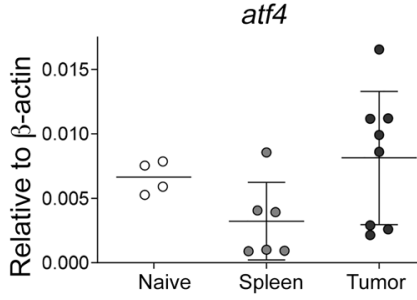

Figure 4. Tumor progression is associated with the elevated ER stress response in PMN-MDSCs. M-MDSCs (A) and PMN-MDSCs (B) were sorted from spleens of naive mice and spleens and tumors of LLC TB mice 3 weeks after tumor inoculation. Gene expression of the key molecules of the different ER stress response pathways was measured; $n=4-10$. Individual values are shown on graphs. $P$ values were calculated using 1-way ANOVA test with correction for multiple comparisons. Values less than 0.05 are shown on graphs.

We asked why deletion of IFN- $\gamma$ R2 in M-MDSCs did not abrogate their suppressive activity in the tumor microenvironment (TME). We hypothesized that other factors present in TME could compensate for the loss of IFN- $\gamma \mathrm{R}$ signaling. To test this hypothesis, M-MDSCs were isolated from spleens of TB IFN- $\gamma \mathrm{R} 2^{\Delta \mathrm{Myel}}$ or control mice and then treated in the presence of tumor explant supernatants for 24 hours in the presence of lactic acid (enriched in TME), dimethyloxallyl glycine (HIF-1 $\alpha$ stabilizing agent), or exposed to $0.1 \%$ hypoxia. M-MDSCs from spleens of control LLC TB mice had potent suppressive activity. This suppression was abrogated in M-MDSCs from IFN- $\gamma \mathrm{R} 2^{\triangle \mathrm{Myyl}} \mathrm{LLC}$ TB mice. Exposure of these cells to hypoxia or lactic acid did not restore suppressive activity of M-MDSCs (Figure 9A). Next, we explored the effect of proinflammatory cytokines present in TME with known activity on MONs. Treatment of splenic IFN- $\gamma \mathrm{R} 2^{\text {Myyel }}$ MONs with a range of concentrations of IL-1 $\beta$, TNF- $\alpha$, IFN- $\beta$, or HMGB1 did not restore suppressive activity of spleen M-MDSCs from IFN- $\gamma \mathrm{R} 2^{\Delta \text { Myel }}$ LLC TB mice (Figure 9B). However, treatment with IL-6 reversed the effect of IFN- $\gamma$ R2 deletion and restored suppressive activity of M-MDSCs (Figure 9B). We measured the presence of IL-6 in spleens and tumors of TB mice and found that IL- 6 in TME was dramatically higher than in spleens (Figure 9C). To investigate the role of IL- 6 in the regulation of tumor progression and tumor M-MDSC function, we neutralized IL-6 signaling with anti-IL-6R antibody. Treatment of LLC TB mice with IL-6R antibody did not significantly reduced tumor growth in WT mice. However, in IFN- $\gamma$ R2 conditional KO mice, it resulted in a substantial decrease in tumor progression (Figure 9D). Suppressive capacity of tumor IFN- $\gamma \mathrm{R}^{\mathrm{\Delta Myel}}$ MONs was significantly attenuated in mice treated with anti-IL-6R antibody. No such effect was observed in WT mice treated with anti-IL-6R antibody (Figure 9E). We asked if the continuous presence of IL- 6 is required to maintain suppressive activity by M-MDSCs. To experimentally address this question, bone marrow progenitors were cultured with GM-CSF and IL- 6 for 3 days and then split into 2 groups. In the first group cells were cultured for additional 3 days in the presence of IL-6. In the second group, cells were cultured for an additional 3 days in the absence of IL-6. IL-6 withdrawal from the culture resulted in loss 
A IRE1 $\alpha^{\text {fl/fl }}$ LysM Cre

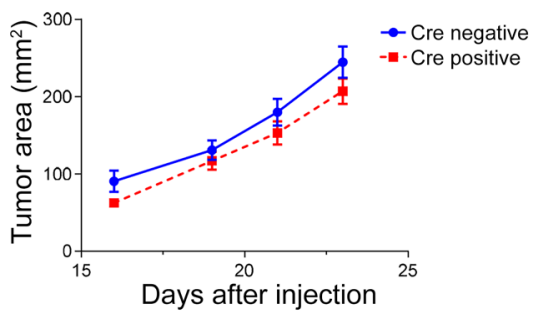

CHOP KO

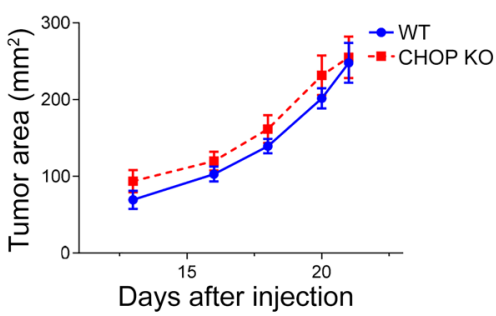

ATF6 $^{\text {flifl LysM Cre }}$

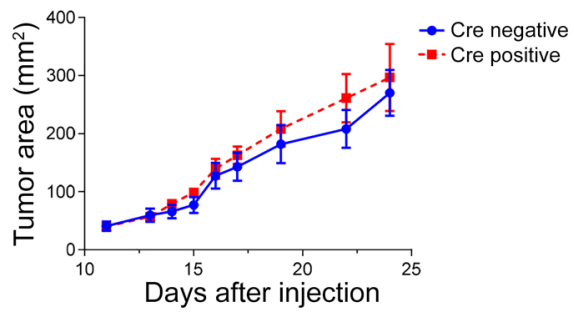

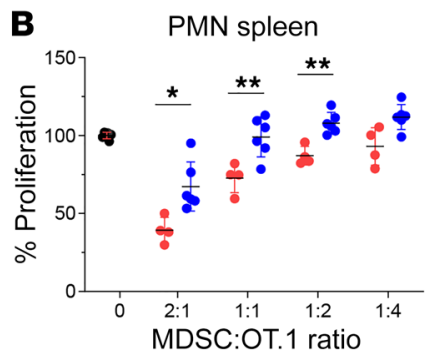
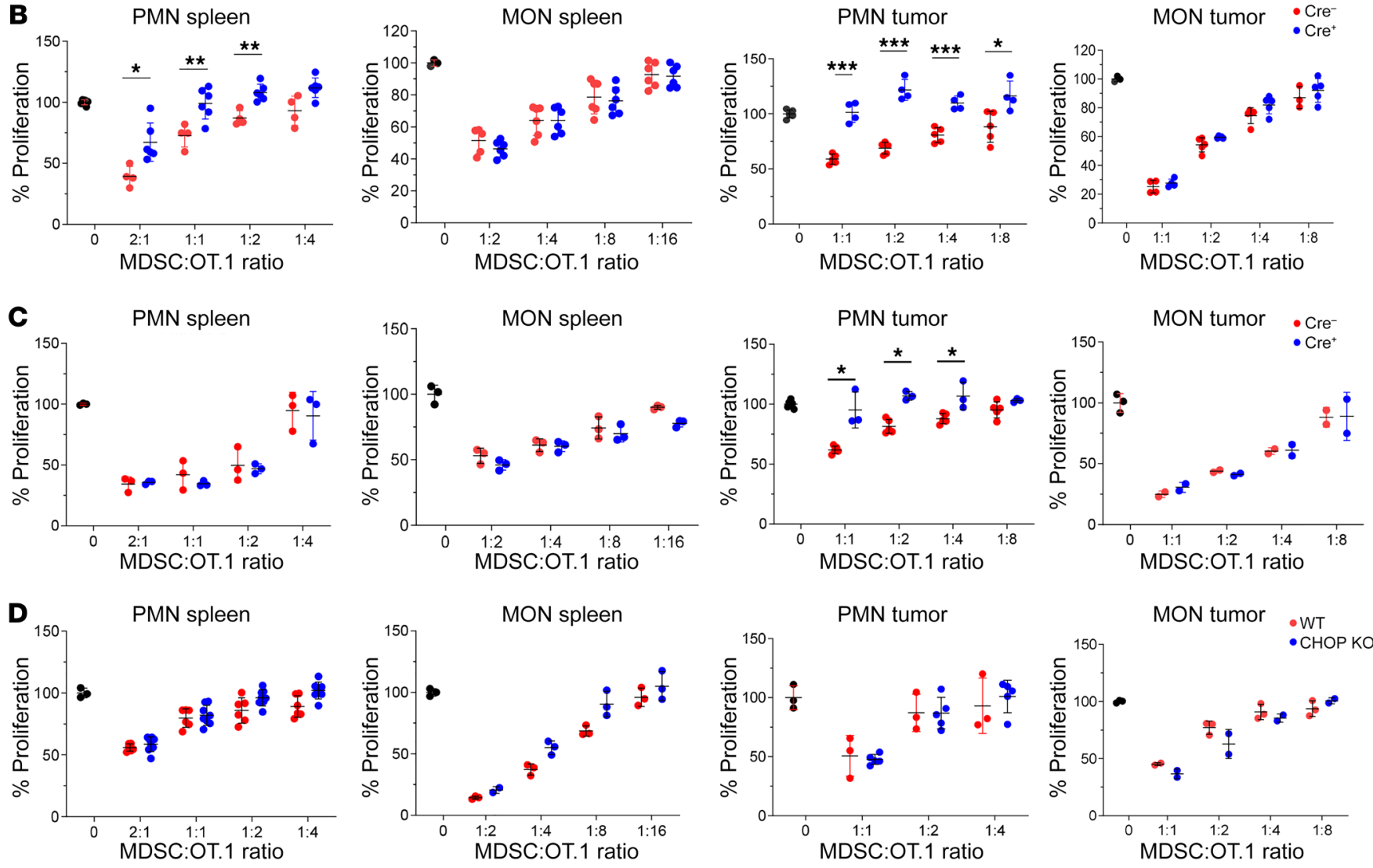

Figure 5. ER stress pathways regulate suppressive activity of PMN-MDSCs, but not M-MDSCs, during tumor progression. Mice with deletions of the specified key molecules of different ER stress response pathways were injected with LLC tumor cells and the kinetics of tumor growth were measured (A). The representative results of 1 of 2 independent experiments are shown; $n=6$ for control and KO mice for IRE $1 \alpha, n=6$ for control and CHOP-KO mice, $n=9$ for control and $n=4$ for ATF6 KO mice. M-MDSCs and PMN-MDSCs were sorted from spleens and tumors of LLC TB IRE1 $\alpha^{\Delta \text { Myel }}$ mice (B), ATF6 $6^{\Delta M y e l}$ mice (C), and CHOP-KO mice (D), and their suppressive activity was evaluated and compared with the cell populations from the control (Cre-) littermates (B and $\mathbf{C})$ or WT (D) mice. The results of 3 independent experiments are shown. ${ }^{*} P<0.05,{ }^{* *} P<0.01$; ${ }^{* * *} P<0.001$ from control in 2 -sided unpaired Student's $t$ tests.

of suppressive activity by MDSCs, whereas in the presence of IL-6 they demonstrated suppressive activity (Figure 9F). We also tested an effect of IL-6 on T cell proliferation directly. Two-day culture of splenocytes with IL-6 did not affect T cell proliferation (Figure $9 \mathrm{G})$. Thus, taken together, these results suggested that IL-6 could be one of the nonredundant mechanisms that support M-MDSC function in tumors.

\section{Discussion}

In this study, we identified specific mechanisms that could govern accumulation of immune-suppressive activity by MDSCs in cancer and chronic viral infection. By comparing the functional activity of M-MDSCs and PMN-MDSCs side by side in different models, we have established that although MDSCs in chronic infection and can- cer shared a number of common features, there were fundamental differences. First, chronic LCMV infection did not cause generation of PMN-MDSCs, whereas in cancer models, both M-MDSCs and PMN-MDSCs were prominent features in both spleens and tumors. Consistent with a previous report, chronic but not acute LCMV infection caused accumulation of M-MDSCs (19). We hypothesized that one of the reasons for accumulation of MDSCs in chronic infection and cancer is a persistent stimulation of myelopoiesis that in combination with proinflammatory factors might lead to activation of the UPR and acquisition of immune-suppressive activity by myeloid cells. A recent study implicating nutrient deprivation sensor GCN2 in immune-suppressive activity of MDSCs involving ER stress pathways supports this hypothesis (20). However, although both LCMV-C13 infection and tumors caused accumulation of 
A

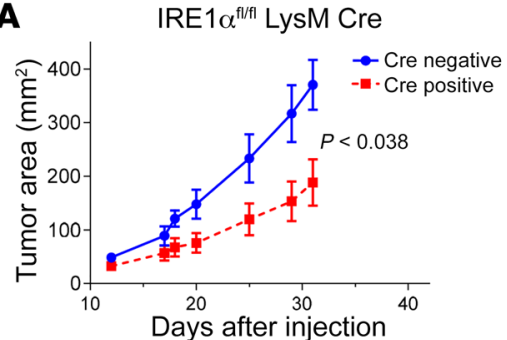

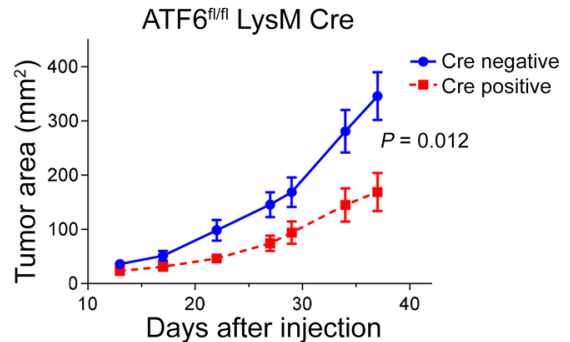

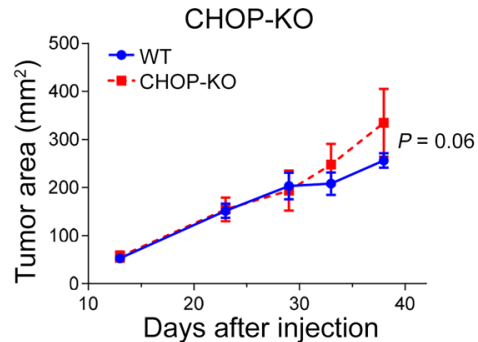

B

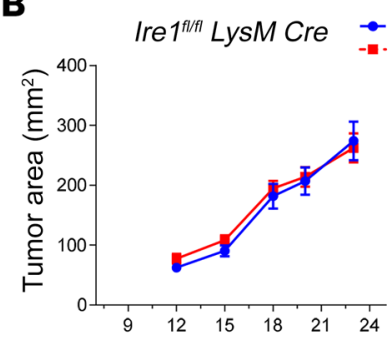

Days after injection
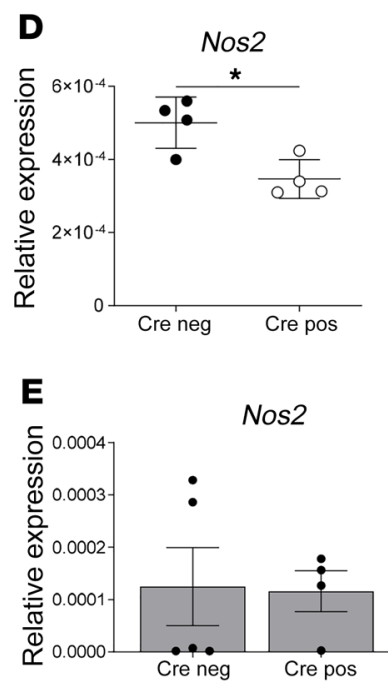

c

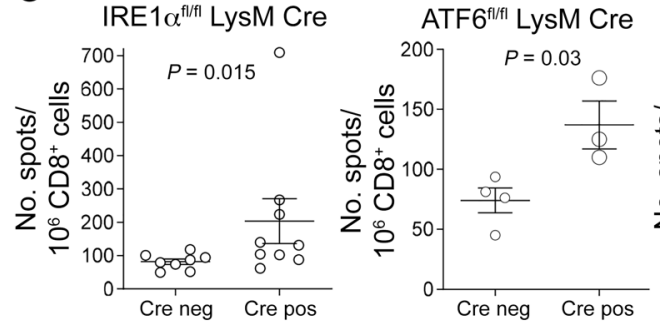

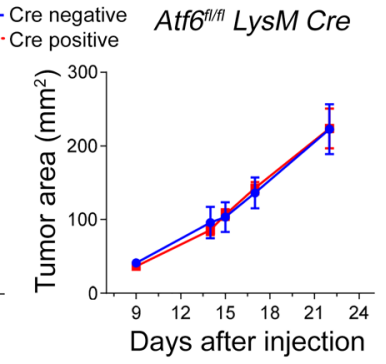

$\operatorname{Arg} 1$
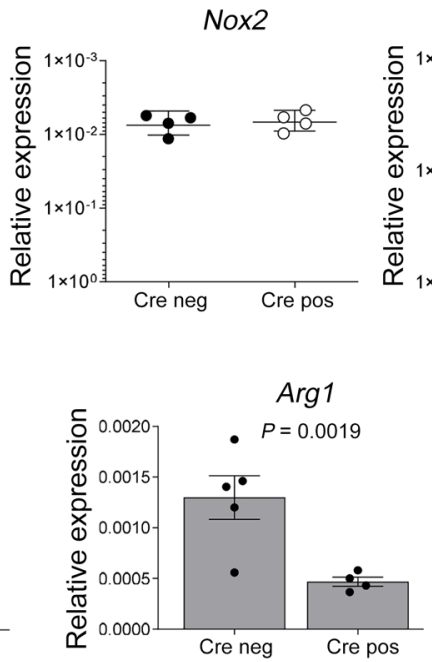

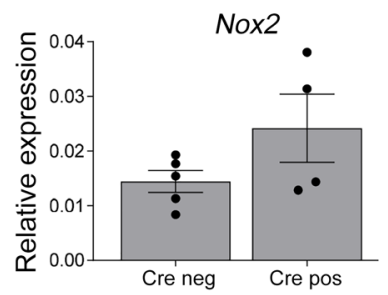

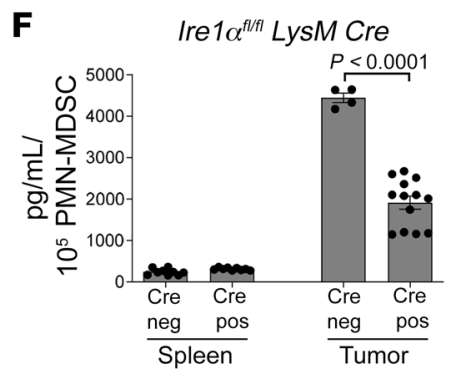

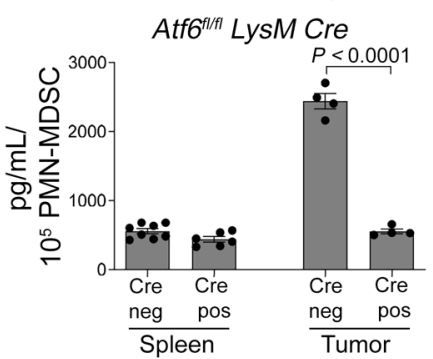

Figure 6. IRE1 $\alpha$ and ATF6 deletion in myeloid cells improves antigen-specific responses and delays the growth of MC38 tumors. (A) MC38 tumor growth in mice with specified deletions. The representative results of 1 of 2 independent experiments are shown; $n=5$ for Cre- control and $n=6$ mice for IRE1 $\alpha^{\Delta \text { Myel }}$ mice, $n=5$ for control and $n=6$ for CHOP-KO mice, $n=19$ for Cre- control, and $n=15$ for ATFG ${ }^{\Delta M y e l}$ mice. $P$ values are from 2-way ANOVA test with adjust-

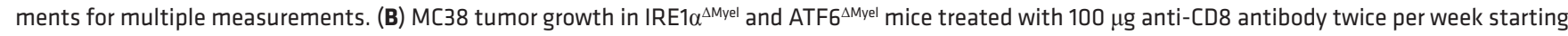
from day 1; $n=8$ for control and $n=4$ for treated IRE1 $\alpha^{\Delta \text { Myel }}$ mice, $n=4$ for control, and $n=4$ for treated ATF6 ${ }^{\Delta \text { Myel }}$ mice. (C) Antigen-specific response to KYMCNSSCM p53 peptide by splenic CD8 ${ }^{+}$T cells from IRE1 $\alpha^{\Delta \text { Myel }}$, CHOP $^{-1-}$, and ATF6 ${ }^{\Delta \text { Myel }}$ mice bearing MC38 tumors was measured by IFN- $\gamma$ ELISPOT; $n=$ 3-8. Individual values are shown on graph. $P$ values calculated with Mann-Whitney $U$ tests are shown on graphs. (D) Expression of genes associated with suppressive activity was evaluated by qRT-PCR in tumor PMN-MDSCs isolated from IRE1 $\alpha^{\triangle M y e l}$ LLC TB mice. The results of 3 independent experiments are shown; $n=4$ and individual values are shown on graphs. ${ }^{*} P<0.05$. (E) Expression of genes associated with suppressive activity was evaluated by $q R T-P C R$ in spleen PMN-MDSCs isolated from IRE1 $\alpha^{\Delta \text { Myel }}$ LLC TB mice. $P$ value was calculated using 2-sided unpaired Student's $t$ tests; $n=4-5$ and individual values are shown on graphs. (F) Spleen and tumor PMN-MDSCs from ATF6 ${ }^{\triangle M y e l}$ MC38 TB CD8 antibody-treated mice with similar tumor sizes and IRE1 $\alpha^{\triangle M y e l}$ LLC TB mice with similar tumor sizes were sorted and cultured for 20 hours. Supernatants were collected and the amount of PGE ${ }_{2}$ was measured by ELISA; $n=$ 4-13. Individual values are shown on graphs. $P$ values calculated from 2-sided Student's $t$ tests are shown.

cells with the phenotypes of PMNs and MONs, immune-suppressive PMN-MDSCs were found only in tumor models. Activation of the ER stress response was not detectable in PMNs or MONs in LCMV-C13 infection. In contrast, it was prominently featured in cancer. One of the possible explanations is that myeloid cell expansion is constantly increased in tumor models over time, whereas in LCMV-13 infection, it peaks around day 14 and then decreases.
This may decrease the pressure on the myeloid compartment and explain the lack of the ER stress response. Alternatively, in cancer there could be other mechanisms able to stimulate the ER stress response. In tumor tissues, hypoxia can be such factor. However, in spleens the mechanism is not clear and is probably regulated by tumor-derived soluble factors. The surprising finding in this study was the fact that although the ER stress response was detected in 
A

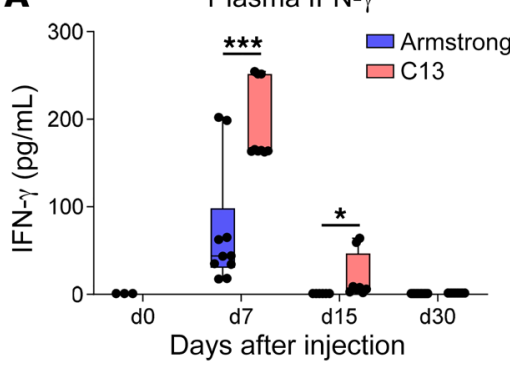

B

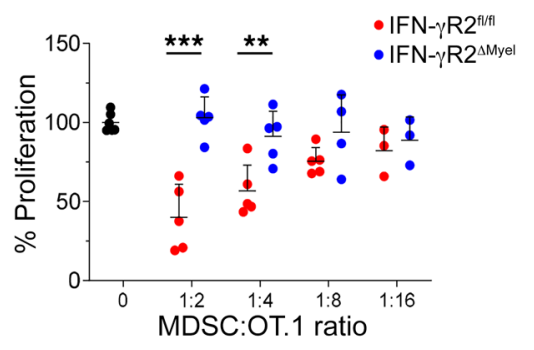

C

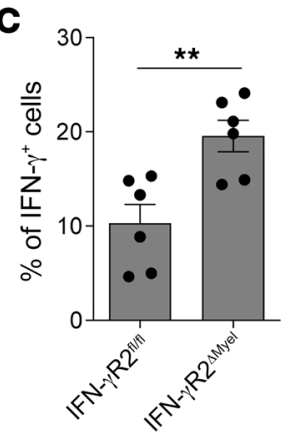

D

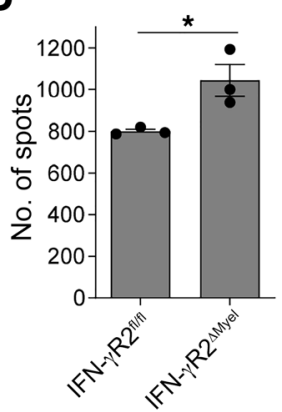

E

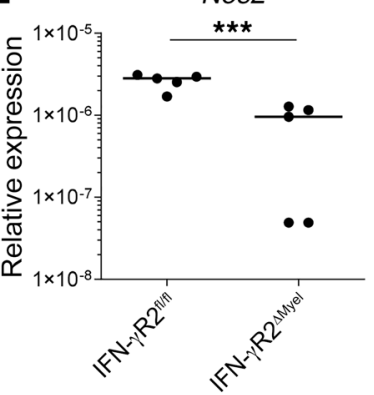

Nox2

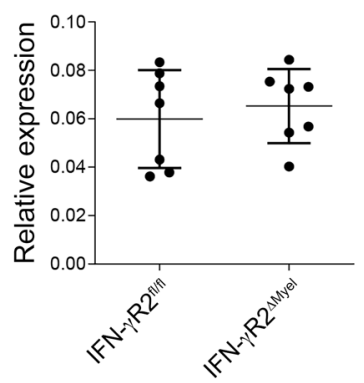

$\operatorname{Arg} 1$

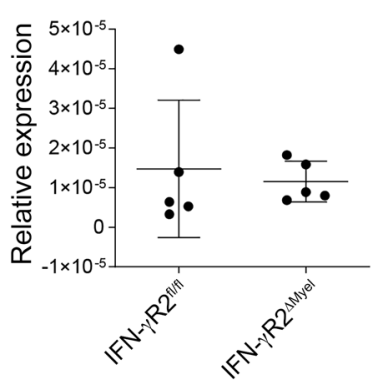

Ptges

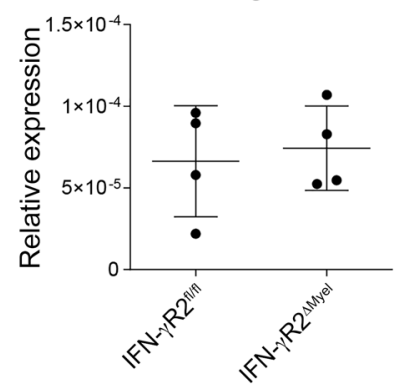

\section{$\mathbf{F}$}

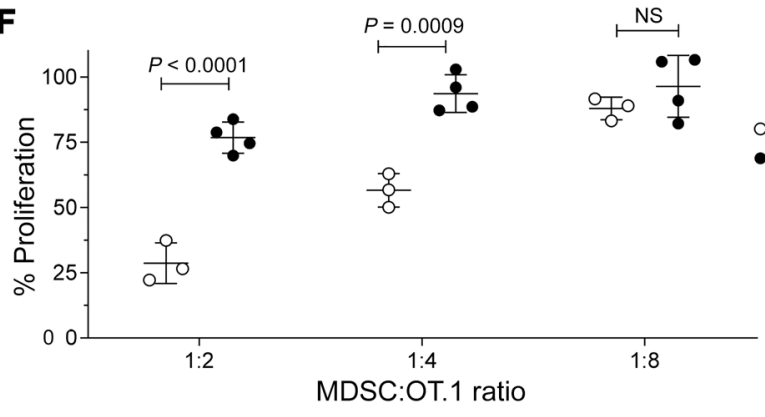

Figure 7. IFN- $\gamma$ regulates suppressive activity of splenic M-MDSCs during chronic LCMV infection. (A) C57BL/6 mice were infected with LCMV-Arm or LCMV-C13 and the concentrations of plasma IFN- $\gamma$ were measured at different time points by ELISA. The results of 2 independent $(n=9)$ experiments are shown. (B-E) IFN- $\gamma R 2^{f / / f I}$ LysM-Cre positive and negative littermate mice (IFN- $\gamma R 2^{\Delta M y e l}$ and IFN- $\gamma R 2^{f / / f I}$, respectively) were infected with LCMV-C13. Monocytic cells were sorted from spleens on day 14 and their suppressive activity was measured in cocultures with activated OT.1 splenocytes (B). Splenic CD8 ${ }^{+}$ T cell responses to specific LCMV-derived peptide (gp33-41) were measured by IFN- $\gamma$ intracellular staining (C) or IFN- $\gamma$ ELISPOT (D). The gene expression of the key immunosuppressive molecules was measured in monocytic cells by qRT-PCR $(\mathbf{E})$. The results of 3 independent experiments are shown $(n=5-6)$. ${ }^{*} P<0.05,{ }^{* *} P<0.01,{ }^{* *} P<0.001$ from control in 2-sided unpaired Student's $t$ tests. (F) Effect of iNOS inhibitor on the suppressive activity of M-MDSCs. Mice were infected with $1 \times 10^{6}$ pfu LCMV C13 strain i.v. and splenic monocytes were sorted on day 14 . Suppression assay with sorted monocytes and OT.1 splenocytes was set up in the presence of vehicle or $700 \mu \mathrm{M} \mathrm{L-NMMA.} \mathrm{Mean} \mathrm{and} \mathrm{SD} \mathrm{are} \mathrm{shown}(n=3-4)$. $P$ values were calculated using Student's $t$ tests.

both MDSC populations in cancer, only PMN-MDSC function was dependent on the ER stress response, whereas it was dispensable for M-MDSC activity. Apparently, other mechanisms more prominently control suppressive activity of M-MDSCs than ER stress.

IRE1 $\alpha$ and ATF6 pathways were directly involved in control of PMN-MDSC suppressive activity in TB mice, since the deletion of these pathways abrogated function of PMN-MDSCs in spleens and tumors. ATF6 signaling was implicated in the adaption to long-term chronic stress $(21,22)$. The $\mathrm{N}$-terminal cytosolic fragment of ATF6 functions as a transcription factor and forms heterodimers with XBP1. Furthermore, ATF6 was shown to regulate transcription of Xbp1 (23) and deletion of ATF6 decreased ER stress-induced BiP expression (24). IRE1 $\alpha$ and ATF6 pathways converge on regulation of Xbp1-dependent signaling (25). Thus, it is possible that this common mechanism may regulate PMN-
MDSC function. Our data for CHOP-targeted deletion were not consistent with a report demonstrating cancellation of MDSC suppressive activity in tumor MDSCs and a resulting antitumor effect (12). A recent study linked activation of CHOP with microbiota (26). It is possible that the effect of CHOP on MDSCs may depend on microbiota present in different facilities.

Our data indicate that in cancer abrogation of PMN-MDSCs, suppressive activity was sufficient to cause a delay of growth of the immunogenic tumor. This effect was mediated by $\mathrm{CD} 8^{+} \mathrm{T}$ cells. These results support the notion that in types of cancer associated with spontaneous immune responses, PMN-MDSCs play an important role in regulation of tumor progression and targeting of PMN-MDSCs may be clinically beneficial.

Our data also indicate that in cancer and chronic LCMV infection, suppressive activity of M-MDSCs was not controlled by the 


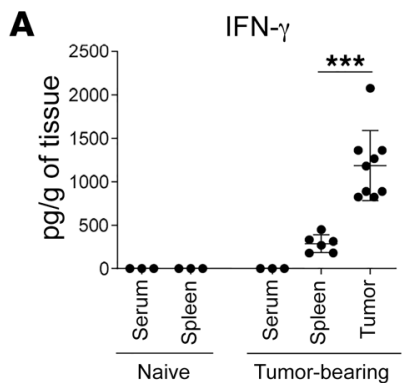

D

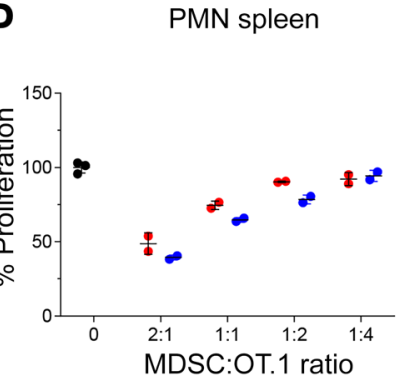

MON spleen

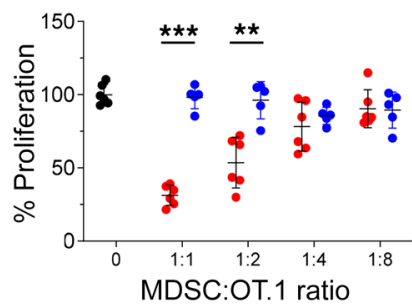

B

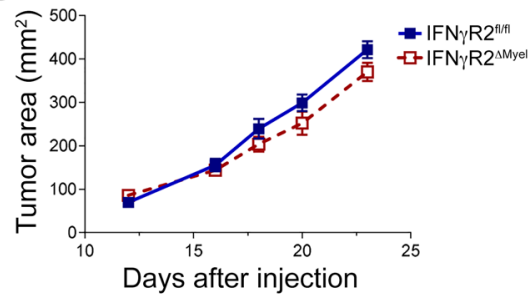

C

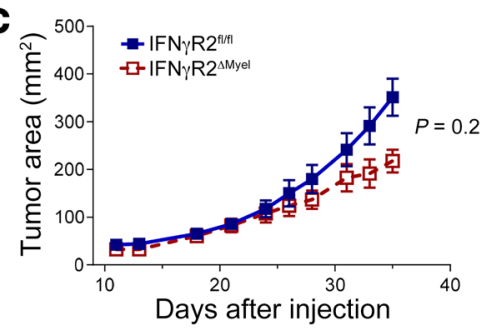

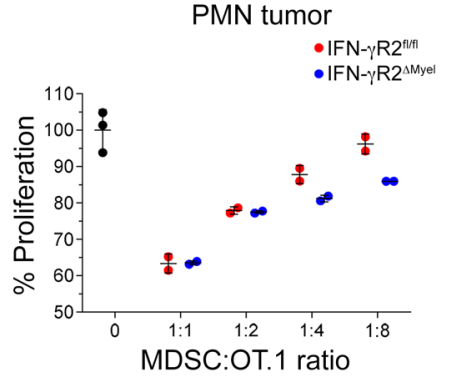

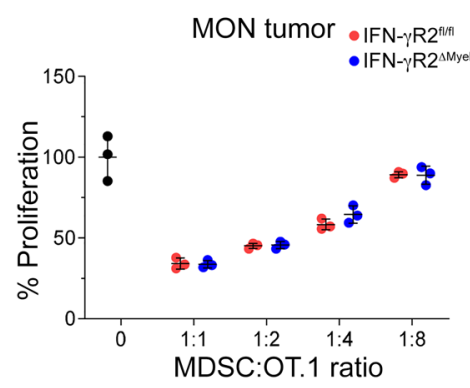

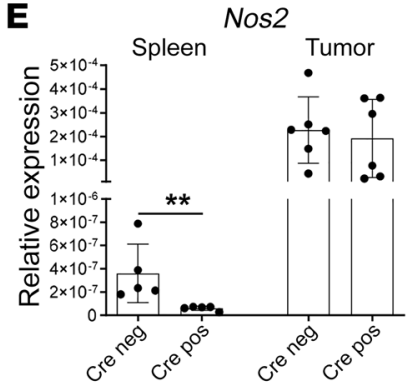
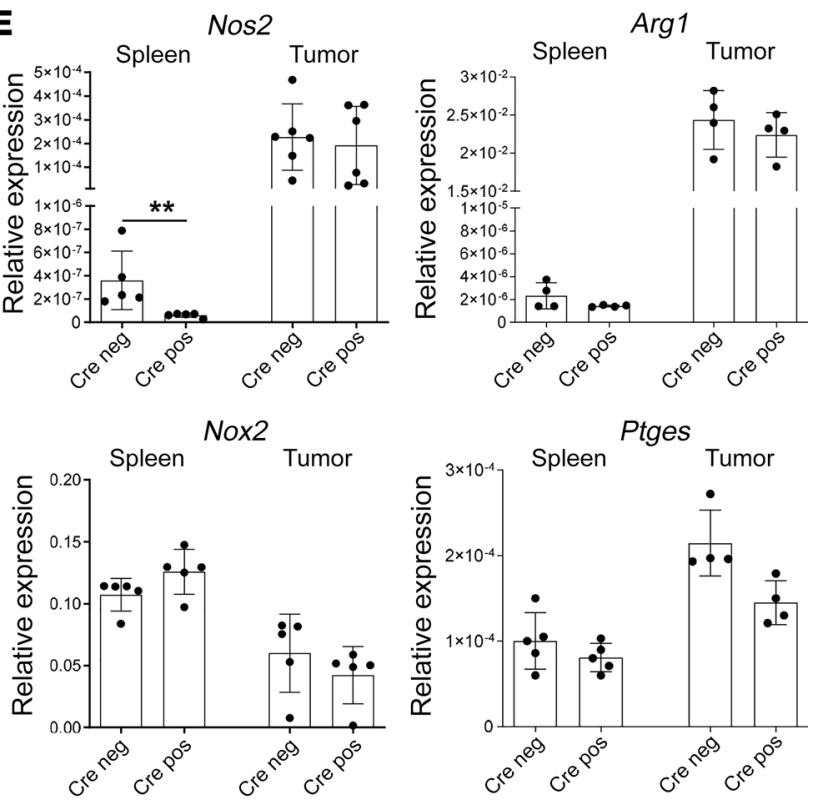

Figure 8. IFN- $\gamma$ regulates suppressive activity of splenic M-MDSCs during tumor progression. (A) IFN- $\gamma$ concentration in sera, spleens, and tumor lysates from naive and LLC TB mice were measured by ELISA. The results of 3 independent experiments are shown $(n=3-8)$. (B and $\mathbf{C})$ IFN- $\gamma \mathrm{R} 2^{\Delta M y e l}$ and IFN- $\gamma R 2^{f / / f I}$ mice were injected with LLC (B) or MC38 (C) tumor cells and the tumor growth kinetics were measured. The representative results of 1 of 3 similar experiments are shown ( $n=9$ for IFN- $\gamma \mathrm{R} 2^{\mathrm{fl} / f \mathrm{fl}}$ and $n=4$ for IFN- $\gamma \mathrm{R} 2^{\Delta \text { Myel }}$ mice in LLC model, $n=6$ for IFN $-\gamma \mathrm{R} 2^{\mathrm{fl} / \mathrm{fl}}$, and $n=4$ for IFN- $\gamma \mathrm{R} 2^{\Delta \mathrm{Myel}}$ mice in MC38 model). M-MDSCs and PMN-MDSCs were sorted from spleens and tumors. Their suppressive activity was measured in cocultures with activated OT.1 splenocytes (D) and the expression of the key immunosuppressive molecules in M-MDSCs was measured by qRT-PCR (E) ( $n=4-5$ from 2 independent experiments). ${ }^{* *} P<0.01 ;{ }^{* *} P<0.001$ from control using 2-sided Student's $t$ tests.

ER stress response, but instead was dependent on IFN- $\gamma$ signaling. IFN- $\gamma$ is an important component of the innate antiviral response and is predominantly produced by NK cells or innate lymphoid type 1 cells $(27,28)$. Signaling via IFN- $\gamma \mathrm{R}$ leads to activation of JAK1 and JAK2, resulting in homodimerization and phosphorylation of STAT1 (29). IFN- $\gamma$ has been shown to induce NO production (30). It also enhances stimulation of the adaptive immune responses by activating antigen-presenting cells (31-33). IFN- $\gamma$ has been shown to "prime" macrophages to release reactive oxygen species (34), increase macrophage receptor-mediated phagocytosis (35), and promote polarization of macrophages to an M1 phenotype (36). However, our findings demonstrate that IFN- $\gamma$ can indirectly induce an immunoregulatory effect of M-MDSCs. Increased expression of Nos 2 and NO production induced by IFN- $\gamma$ can promote immune-suppressive activity of these cells. Consistent with our observation, the ability of IFN- $\gamma$ to support differentiation of MDSCs in vitro has been reported (37). In vitro treatment of PMN-MDSCs and M-MDSCs generated during M. bovis chronic inflammation with IFN- $\gamma$ promoted PMN-MDSC suppression via upregulation of reactive oxygen species and arginase I, whereas treatment of M-MDSCs reduced their suppressing activity by decreasing the arginase I activity (38). Our data using blockade of IFN- $\gamma$ signaling in myeloid cells in vivo demonstrated that IFN- $\gamma$ is dispensable for suppressive activity of PMN-MDSCs and promotes suppressive activity of M-MDSCs. Our data are consistent with the report showing that the deletion of IFN- $\gamma \mathrm{R}$ in myeloid cells abrogated suppressive activity of MDSCs via NO (39). Deletion of IFN- $\gamma$ R in myeloid cells abrogated M-MDSC suppressive activity in spleens but not in the tumor site, and it did not result in significant antitumor effect. It appears that in the tumor site other factors compensated for the loss of IFN- $\gamma \mathrm{R}$ signaling. We found that one of the factors could be IL-6. The level of IL- 6 inside tumors is much higher than that in spleens. IL-6 triggers different signaling pathways than IFN- $\gamma$ R primarily via STAT3 and was previously directly implicated in MDSC activity (40). STAT3 itself was one of the major transcription factors involved in MDSC accumulation (41). Thus, IL- 6 and IFN- $\gamma \mathrm{R}$ may represent nonredundant mechanisms of M-MDSC regulation. For PMN-MDSCs the 
A

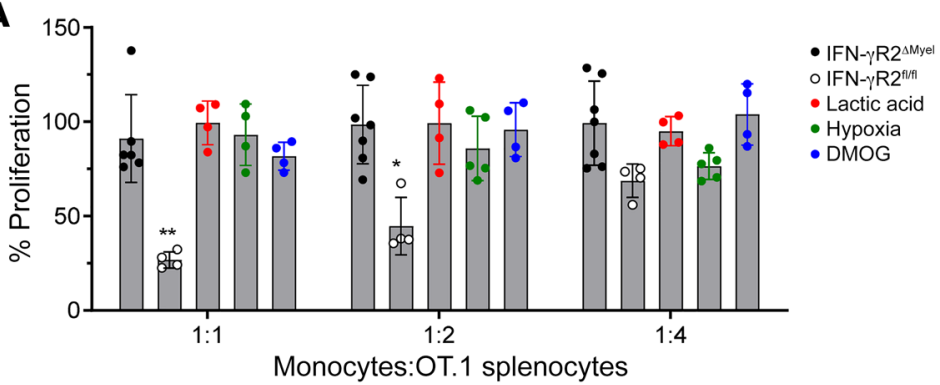

B

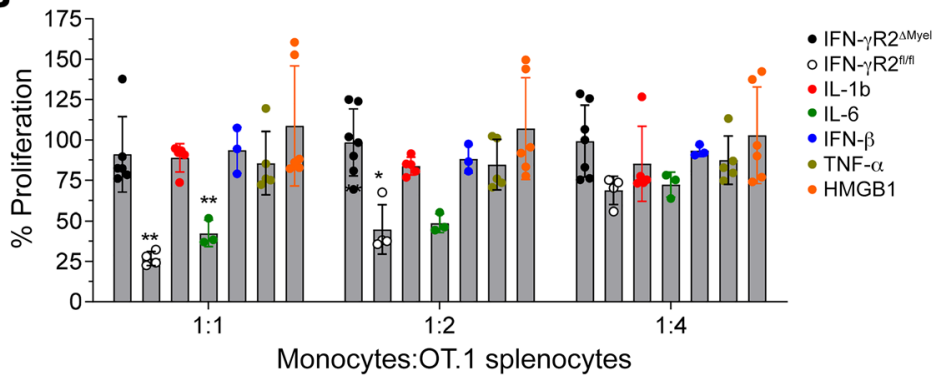

C

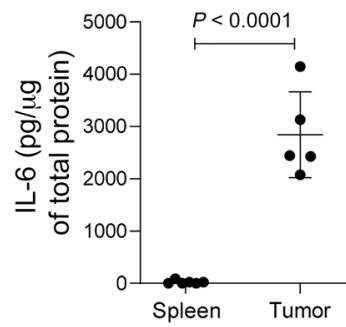

D

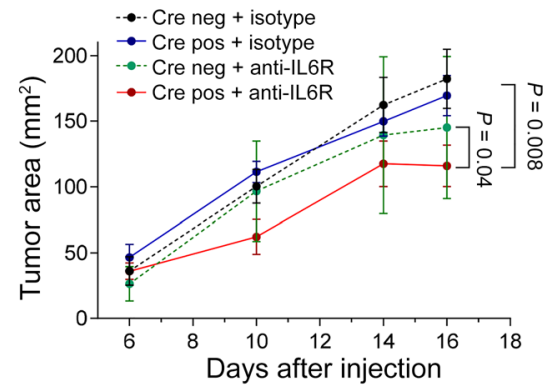

E

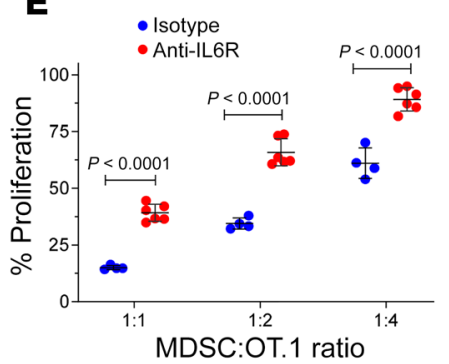

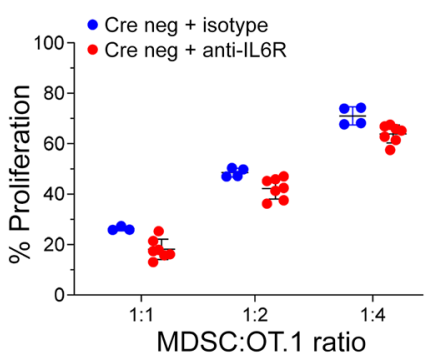
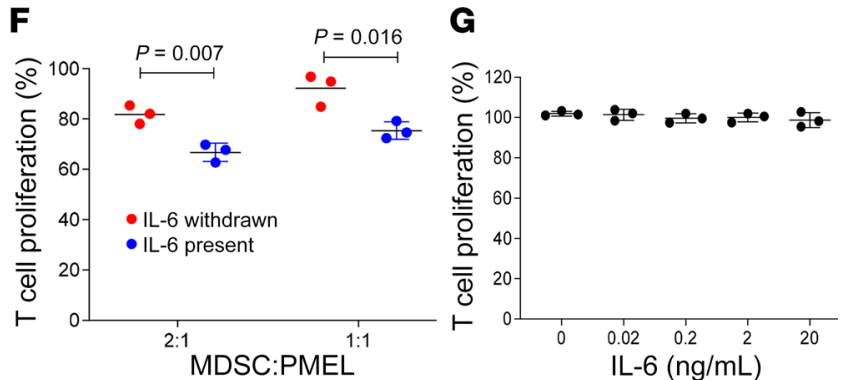

Figure 9. Effect of TME on immune-suppressive activity of M-MDSCs with deleted IFN- $\gamma$ R2. (A and B) Splenic M-MDSCs from LLC TB mice were sorted and cultured in the specified conditions for 24 hours. Their suppressive activity was measured with activated OT.1 splenocytes. The results of 3 independent experiments are shown; $n=3-5$. (C) IL-6 protein concentrations in spleens and tumor lysates from LLC TB mice were measured by ELISA $(n=5)$. $P$ value was calculated using 2-sided unpaired Student's $t$ tests. (D and E) LLC TB mice were treated with $400 \mu \mathrm{g} / \mathrm{mL}$ anti-IL6R or isotype control starting from day 3 every 3 days until day 16. (D) Kinetics of tumor growth were measured. Mean and SD ( $n=6-10$ per group) are shown. $P$ values were calculated using 2-way ANOVA test. (E) Tumor monocytes from IFN- $\gamma$ R2 ${ }^{\Delta \text { Myel }}$ mice (left panel) or WT mice (right panel) ( $n=4$ isotype-treated group, $n=6$ IL-6R antibodytreated group) were sorted and suppressive activity was measured in triplicates with OT.1 splenocytes using ${ }^{3} \mathrm{H}$-thymidine incorporation. Proportion of proliferation from control values (splenocytes stimulated in the absence of MDSCs) is shown. $P$ values were calculated using Student's $t$ test. (F) Inhibition of T cell proliferation by MDSCs generated from HPCs. Bone marrow HPCs were cultured for 3 days with GM-CSF and IL-6, then medium was replaced with a new medium with or without IL-6. Cells were collected 3 days later and incubated with splenocytes from PMEL mice in the presence of $10 \mathrm{ng} / \mathrm{mL}$ cognate gp100-derived peptide at 2:1 and 1:1 ratios. Cell proliferation was measured after 48 hours; $n=3$. $P$ values were calculated using unpaired 2-sided Student's $t$ tests. (C) Splenocytes isolated from PMEL mice were cultured with $10 \mathrm{ng} / \mathrm{mL}$ cognate gp100-derived peptide in the presence of different concentrations of IL-6 (0.02-20 ng/mL). Proliferation of T cells was measured after 48 hours.

situation is different, since their functional activity is controlled by the ER stress response. In tumors, the ER stress response can be further enhanced as compared with spleens, probably because of hypoxia. Therefore, the blockade of the ER stress response in PMN-MDSCs abrogated their suppressive activity.

Thus, our data indicate that the mechanisms of MDSC activation are different in PMN-MDSCs and M-MDSCs. The ER stress response appears to be a dominant factor in regulating PMN-MDSCs and at least in one immunogenic tumor model is enough to reduce tumor progression. In combination with a number of reports in recent years demonstrating the critical role of PMN-MDSCs in tumor progression (42-44), these results open an opportunity to selectively target these cells. In contrast, M-MDSCs did not depend on activation of the ER stress response but rather on IFN- $\gamma$ R signaling. Since IFN- $\gamma$ production is associated with potent immune response in cancer or infections, this mechanism may serve to limit the extent of $\mathrm{T}$ cell activation. Thus, an effective approach to immune therapy may require specific targeting of different immune suppression regulation mechanisms.

\section{Methods}

Mice. C57BL/6 mice (male and female, 6-10 weeks old) were obtained from Charles River or from Envigo. All genetically engineered mice were on C57BL/6 background. CHOP-KO mice were obtained from The Jackson Laboratory. Mice with floxed exons 16 to 17 in the Ire1 $\alpha$ gene were originally generated by the Randal Kaufman group (Sanford Brigham Prebys, La Jolla, California, USA) and Ire1 $\alpha / L y s M$-cre mice were obtained from Deyu Fang (Northwestern University, Chicago, 
Illinois, USA). Mice with the floxed exons 8 to 9 in Atf6 gene were originally generated by the Gokhan Hotamisligil group (Harvard University, Boston, Massachusetts, USA) and obtained from The Jackson Laboratory. They were crossed to LysM-cre mice obtained from The Jackson Laboratory. Ifngr $2^{f l} /$ Lys $M$-cre mice were generated as described (45) using C57Bl6 Agouti ES cell line Ifngr ${ }^{\text {tmla(KOMP)Wtsi }}$ generated in The Knockout Mouse Project (KOMP, catalog 057086-UCD, https://www. mmrrc.org/catalog/cellLineSDS.php?mmrrc_id=57086; International Mouse Phenotype Project, https://www.mousephenotype.org/data/ alleles/MGI:107654/tm1a\%2528KOMP\%2529Wtsi). After the deletion of neo cassette using in vivo $\beta$-actin FLP deleter, an IFN- $\gamma$ R2 floxed strain was established and further crossed to LysM-Cre strain. Mice were routinely genotyped for floxed, WT, or residual deleted allele by PCR using the following primers: ACTGCTTGGCTGTGACTGATT, CCATGTGGGATGGTTCCGTT, CCTGGTGCAGCAAACCCTAT. The presence of WT allele resulted in a $172 \mathrm{bp}$ band, floxed allele (388 $\mathrm{bp})$, and deletion of the floxed allele was detected by $465 \mathrm{bp}$ band.

Antibodies and cell lines. A list of antibodies used in the study is provided in Supplemental Table 1. Murine lung cancer cell line LLC and lymphoma cell line EL-4 were purchased from ATCC. MC38 was obtained from Kerafast. All cells were routinely tested for mycoplasma and found to be free of contamination.

Viral infection, tumor growth experiments, and cell isolation. In viral experiments, mice were injected i.v. with $1 \times 10^{6}$ pfu Armstrong or C13 strains of LCMV. For tumor growth experiments, mice were injected s.c. with $3 \times 10^{5}$ EL4 thymoma, $3 \times 10^{5}$ LLC, or $8 \times 10^{5}$ MC38 colon carcinoma tumor cells. Mice were sacrificed at specified time points, and spleens and/or tumors were isolated. Spleens were homogenized through a $70 \mu \mathrm{m}$ filter and red blood cells were lysed by ACK buffer. Total tumor cells were isolated with the use of tumor dissociation kit (Miltenyi). Splenic PMNs (Ly6G+) and CD8 ${ }^{+}$T cells were pulled down by magnetic beads (Miltenyi). Splenic and tumor monocytes (CD $45^{+}$

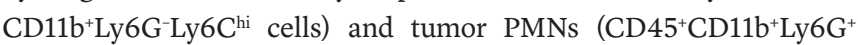
Ly6C ${ }^{\text {int }}$ cells) were sorted by FACS Aria II (Becton Dickinson).

Suppressive assays. PMNs or MONs were cocultured in 96-well round-bottom plates with the mixture of OT.1 and WT splenocytes at a $1: 4$ ratio in the presence of $0.5 \mathrm{ng} / \mathrm{mL}$ SIINFEKL peptide (specific to OT.1 receptor). T cell proliferation was assessed by ${ }^{3} \mathrm{H}$-thymidine incorporation using TopCount machine (PerkinElmer).

Quantitative real-time PCR. Total RNA was extracted with total RNA microkit (Zymo) according to the manufacturer's protocol. Complementary DNA (cDNA) was synthesized with the use of a High-Capacity cDNA Reverse Transcription Kit (AppliedBiosystems). qRT-PCR was performed using Power SYBR Green PCR Master Mix (Applied Biosystems) and QuantStudio 6 Flex Real-Time PCR System (Applied Biosystems). Primers are described in Supplemental Table 2.

ELISA. IFN- $\gamma$ and IL- 6 were measured in blood plasma, spleen, and tumor lysates using Ready-Set-Go ELISA kits (Thermo Fisher Scientific). Prostaglandin $\mathrm{E}_{2}$ was measured in cell supernatants with the commercial kit from Thermo Fisher Scientific.

Flow cytometry and Western blotting. Flow cytometry for specified markers was conducted with the use of a BD LSR II flow cytometer (Becton Dickinson). Intracellular staining was performed with a BD Cytofix/ Cytoperm kit used according to the manufacturer's recommendations.

For Western blotting, whole cell extracts were fractionated by SDS-PAGE and transferred to a polyvinylidene difluoride membrane using a transfer apparatus according to the manufacturer's protocols
(Bio-Rad). After blocking with 5\% nonfat milk in PBST (PBS, pH 7.5, $0.5 \%$ Tween 20) for 60 minutes, the membrane was washed once with PBST and incubated for 2 hours with primary antibodies followed by a 1 hour incubation with the corresponding secondary antibody and ECL development (Amersham Biosciences).

ELISPOT assays. To evaluate the in vivo presence of $\mathrm{T}$ cells specific to LCMV-derived gp33-41 KAVYNFATM peptide or p53-derived KYMCNSSCM overexpressed by MC38 tumor cells, IFN- $\gamma$ ELISPOT assay was performed. Briefly, $\mathrm{CD} 8^{+} \mathrm{T}$ cells were isolated from the spleens using magnetic beads (Miltenyi). The same number of isolated CD $8^{+} \mathrm{T}$ cells was mixed with $4 \times 10^{5}$ irradiated total WT naive splenocytes and restimulated with $2 \mu \mathrm{g} / \mathrm{mL}$ (KAVYNFATM) or $5 \mu \mathrm{g} / \mathrm{mL}$ (KYMCNSSCM) peptide. Cells were cultured for 48 hours in the PVDF membrane plates (MilliporeSigma) precoated with anti-IFN- $\gamma$ capture antibody (Mabtech), and then the plates were washed and developed according to the manufacturer's procedure (Mabtech).

Cytokine quantification in tissues. Tumors and spleens were collected from LLC TB mice on days 17 to 20 and were mechanically homogenized by FastPrep-24 5G (MP Biomedicals) in 0.5 mL RIPA buffer (Sigma-Aldrich) containing 1/100 dilution of protease inhibitors (Sigma-Aldrich). IL-6 in lysates were quantified using the ELISA (R\&D Systems). The total protein in lysate was measured by BCA protein assay (Pierce) and the amount of cytokine in tissue was normalized to total protein.

Generation of MDSCs from hematopoietic progenitor cells (HPCs). Bone marrow HPCs were enriched with the Miltenyi enrichment kit and cultured with $40 \mathrm{ng} / \mathrm{mL}$ GM-CSF and $20 \mathrm{ng} / \mathrm{mL}$ IL- 6 for 3 days. Medium was replaced with a new one with or without IL-6. Three days later $\mathrm{Gr}-1^{+}$cells were isolated and evaluated in immune suppression assay.

RNA-sequencing. Splenocytes were stained with fluorophoreconjugated antibodies in Brilliant Stain Buffer (BD Bioscience) on ice for 30 minutes in the dark. The surface-stained cells were washed twice with RNase-free staining buffer ( $1 \times$ D-PBS, 0.5\% BSA [MP Biochemical], 0.4 U/ $\mu \mathrm{L}$ RNasin Plus RNase Inhibitor [Promega]) at $400 \mathrm{~g}$ for 5 minutes and then fixed with $1 \times$ Foxp 3 Fixation/Permeabilization working solution for 30 minutes at $2^{\circ} \mathrm{C}$ to $8^{\circ} \mathrm{C}$ in the dark. The fixed cells were washed (not resuspended) twice with ice-cold RNasefree staining buffer without breaking the pellet for complete removal of fixation buffer. The cell pellet was resuspended to the concentration $2 \times 10^{7} / \mathrm{mL}$ to $3 \times 10^{7} / \mathrm{mL}$ in ice-cold RNase-free staining buffer, filtered with $70 \mu \mathrm{m}$ nylon mesh, and immediately CD $45^{+} \mathrm{CD} 11 \mathrm{~b}^{+}$ Ly6 $\mathrm{C}^{+} \mathrm{Ly} 6 \mathrm{G}^{-}$cells (M-MDSCs) were sorted by BD FACSAria Fusion Cell Sorter (BD Bioscience) using $100 \mu \mathrm{m}$ nozzle. The sorted cell pellet was snap-frozen and kept at $-80^{\circ} \mathrm{C}$ until RNA extraction. Total RNA was extracted from 1 to 2 million M-MDSCs using the miRNeasy FFPE RNA Isolation kit (Qiagen). RNA quantity was determined using the Qubit 3.0 Fluorometer (Thermo Fisher Scientific) and the quality was validated using the TapeStation High Sensitivity RNA ScreenTape (Agilent). A quantity of 500 ng DNAse I-treated total RNA was used to prepare the library for Illumina Sequencing using the Quant-Seq 3'mRNA-Seq Library Preparation Kit (Lexogen). Library quantity was determined using qPCR (KAPA Biosystem). Overall library size was determined using the Agilent TapeStation and the DNA High Sensitivity D5000 ScreenTape (Agilent). Equimolar amounts of each sample library were pooled and denatured in a high-output, single-read 75 bp cycle. Next Generation Sequencing was done on a NextSeq 500 (Illumina). 
Reads were mapped to Mus musculus genome (mm10) genome using Star (46) for each sample. Uniquely mapped reads were counted using htseq-count (https://htseq.readthedocs.io/en/master/count. html) and normalized with size factors using DESeq2 (47). Differentially expressed genes across conditions were identified using DESeq2 (47) with the cutoffs abs $\left(\log _{2}\right.$ FC) greater than 1 and adjusted $P$ value less than 0.05. Gene set enrichment analysis were performed using fGSEA R package (https://doi.org/10.1101/060012) with hallmark gene sets from mouse MSigDB (48). Pathway enrichment analysis of differentially expressed genes were conducted using g:Profiler (49). Sequences were deposited to the GEO database, accession number GSE179278.

Statistics. Statistical analyses were performed using 2-tailed Student's $t$ test or Mann-Whitney $U$ test and GraphPad Prism 8.4.2 software (GraphPad Software Inc.). In experiments with more than 2 variables, statistical analysis was performed using ANOVA test with correction for multiple variables. Tumor growth with repeated tumor measurements was evaluated using 2-way ANOVA test. All the data are mean \pm SEM and a $P$ value less than 0.05 is considered significant.

Study approval. Mouse experiments were approved by the IACUC of The Wistar Institute and AstraZeneca.

\section{Author contributions}

ENT performed experiments and wrote the manuscript. SH performed experiments and analyzed data. AH, DC, CC, AL, FP, SH, $\mathrm{BD}, \mathrm{KCH}$, and $\mathrm{KM}$ performed experiments. GK and TWFS analyzed data. CHAT performed experiments and edited the manuscript. CCAH participated in the design of experiments and edited the manuscript. WG provided materials. SG provided materials and edited the manuscript. MAAA and JCB performed experiments and provided materials. EJW participated in study design, provided materials, and edited the manuscript. YN participated in data analysis, provided materials, and edited the manuscript. DIG designed the experiments, analyzed data, and wrote the manuscript.

\section{Acknowledgments}

We thank Randal Kaufman (Sanford Brigham Prebys) and Deyu Fang (Northwestern University) for providing IRE1 $\alpha \times$ LysM-Cre mice, and the AstraZeneca Production Informatics team for performing RNAseq read alignment and qualification. This work was supported by NIH grants R01CA100062, RO1CA216936, R01CA163910, R01CA227629, and CA218133, and the Wistar Institute Animal and Flow cytometry core facilities under Cancer Center support grant P30 CA010815.

Address correspondence to: Dmitry I. Gabrilovich, AstraZeneca, One Medimmune Way, Gaithersburg, Maryland 20878 USA. Email: dmitry.gabrilovich@astrazeneca.com.

SG's present address is: Cedars-Sinai Medical Center, Departments of Medicine and Biomedical Sciences, Los Angeles, California, USA.

CHAT's and CCAH's present address is: Houston Methodist Cancer Center, Houston Methodist Academic Institute, Houston, Texas, USA.
1. Veglia F, et al. Myeloid-derived suppressor cells coming of age. Nat Immunol. 2018;19(2):108-119.

2. Schrijver IT, et al. Myeloid-derived suppressor cells in sepsis. Front Immunol. 2019;10:327.

3. Penaloza HF, et al. The role of myeloid-derived suppressor cells in chronic infectious diseases and the current methodology available for their study. J Leukoc Biol. 2019;105(5):857-872.

4. Safarzadeh E, et al. Myeloid-derived suppressor cells: Important contributors to tumor progression and metastasis. J Cell Physiol. 2018;233(4):3024-3036.

5. Gabrilovich DI. Myeloid-derived suppressor cells. Cancer Immunol Res. 2017;5(1):3-8.

6. Bronte V, et al. Recommendations for myeloid-derived suppressor cell nomenclature and characterization standards. Nat Commun. 2016;7:12150.

7. Condamine T, et al. Lectin-type oxidized LDL receptor-1 distinguishes population of human polymorphonuclear myeloid-derived suppressor cells in cancer patients. Sci Immunol. 2016;1(2):aaf8943.

8. Mastio J, et al. Identification of monocyte-like precursors of granulocytes in cancer as a mechanism for accumulation of PMN-MDSCs. JExp Med. 2019;216(9):2150-2169.

9. Gato M, et al. Drafting the proteome landscape of myeloid-derived suppressor cells. Proteomics. 2016;16(2):367-378.

10. Veglia F, et al. Myeloid-derived suppressor cells in the era of increasing myeloid cell diversity [published online February 1, 2021]. Nat Rev Immunol. https://doi.org/10.1038/s41577-020-00490-y.
11. Condamine T, et al. ER stress regulates myeloid-derived suppressor cell fate through TRAIL-R-mediated apoptosis. JClin Invest. 2014;124(6):2626-2639.

12. Thevenot PT, et al. The stress-response sensor chop regulates the function and accumulation of myeloid-derived suppressor cells in tumors. Immunity. 2014;41(3):389-401.

13. Nan J, et al. Endoplasmic reticulum stress induced $\mathrm{LOX}-1^{+} \mathrm{CD} 15^{+}$polymorphonuclear myeloid-derived suppressor cells in hepatocellular carcinoma. Immunology. 2018;154(1):144-155

14. Yoshida H. ER stress and diseases. FEBS J. 2007;274(3):630-658.

15. Holcik M, Sonenberg N. Translational control in stress and apoptosis. Nat Rev Mol Cell Biol. 2005;6(4):318-327.

16. Ron D, Walter P. Signal integration in the endoplasmic reticulum unfolded protein response. Nat Rev Mol Cell Biol. 2007;8(7):519-529.

17. Claudio N, et al. Mapping the crossroads of immune activation and cellular stress response pathways. EMBO J. 2013;32(9):1214-1224.

18. Wherry EJ, et al. Antigen-independent memory CD8 T cells do not develop during chronic viral infection. Proc Natl Acad Sci US A. 2004;101(45):16004-16009.

19. Norris BA, et al. Chronic but not acute virus infection induces sustained expansion of myeloid suppressor cell numbers that inhibit viral-specific T cell immunity. Immunity. 2013;38(2):309-321.

20. Halaby MJ, et al. GCN2 drives macrophage and MDSC function and immunosuppression in the tumor microenvironment. Sci Immunol. 2019;4(42):eaax8189.

21. Wu J, et al. ATF6alpha optimizes long-term endoplasmic reticulum function to protect cells from chronic stress. Dev Cell. 2007;13(3):351-364.

22. Shoulders MD, et al. Stress-independent activation of XBP1s and/or ATF6 reveals three functionally diverse ER proteostasis environments. Cell Rep. 2013;3(4):1279-1292.

23. Lee $\mathrm{K}$, et al. IRE1-mediated unconventional mRNA splicing and S2P-mediated ATF6 cleavage merge to regulate XBP1 in signaling the unfolded protein response. Genes Dev. 2002;16(4):452-466.

24. Yamamoto K, et al. Transcriptional induction of mammalian ER quality control proteins is mediated by single or combined action of ATF6alpha and XBP1. Dev Cell. 2007;13(3):365-376.

25. Jonikas MC, et al. Comprehensive characterization of genes required for protein folding in the endoplasmic reticulum. Science. 2009;323(5922):1693-1697.

26. Nakamura K, et al. Antibiotic pretreatment alleviates liver transplant damage in mice and humans. JClin Invest. 2019;129(8):3420-3434.

27. Karupiah G, et al. Inhibition of viral replication by interferon-gamma-induced nitric oxide synthase. Science. 1993;261(5127):1445-1448.

28. Weizman OE, et al. ILC1 confer early host protection at initial sites of viral infection. Cell. 2017;171(4):795-808.

29. Gotthardt D, Sexl V. STATs in NK-cells: the good, the bad, and the ugly. Front Immunol. 2016;7:694.

30. Croen KD. Evidence for antiviral effect of nitric 
oxide. Inhibition of herpes simplex virus type 1 replication. J Clin Invest. 1993;91(6):2446-2452.

31. Goldszmid RS, et al. NK cell-derived interferon- $\gamma$ orchestrates cellular dynamics and the differentiation of monocytes into dendritic cells at the site of infection. Immunity. 2012;36(6):1047-1059.

32. Martin-Fontecha A, et al. Induced recruitment of NK cells to lymph nodes provides IFN-gamma for T(H)1 priming. Nat Immunol. 2004;5(12):1260-1265.

33. Schroder K, et al. Interferon-gamma: an overview of signals, mechanisms and functions. J Leukoc Biol. 2004;75(2):163-189.

34. MacMicking J, et al. Nitric oxide and macrophage function. Annu Rev Immunol. 1997;15:323-350.

35. Drevets DA, et al. Complement receptor type 3 mediates phagocytosis and killing of Listeria monocytogenes by a TNF-alpha- and IFNgamma-stimulated macrophage precursor hybrid. Cell Immunol. 1996;169(1):1-6.

36. Kang K, et al. Interferon- $\gamma$ represses M2 gene expression in human macrophages by disassembling enhancers bound by the transcription factor
MAF. Immunity. 2017;47(2):235-250.

37. Ribechini E, et al. Novel GM-CSF signals via IFN- $\gamma$ R/IRF-1 and AKT/mTOR license monocytes for suppressor function. Blood $A d v$. 2017;1(14):947-960.

38. Zhan X, et al. IFN- $\gamma$ differentially regulates subsets of $\mathrm{Gr}-1(+) \mathrm{CD} 11 \mathrm{~b}(+)$ myeloid cells in chronic inflammation. Mol Immunol. 2015;66(2):451-462.

39. Forghani $P$, et al. Properties of immature myeloid progenitors with nitric-oxide-dependent immunosuppressive activity isolated from bone marrow of tumor-free mice. PLoS One. 2013;8(7):e64837.

40. Marigo I, et al. Tumor-induced tolerance and immune suppression depend on the $\mathrm{C} / \mathrm{EBPb}$ ta transcription factor. Immunity. 2010;32(6):790-802.

41. Condamine $\mathrm{T}$, et al. Transcriptional regulation of myeloid-derived suppressor cells. J Leukoc Biol. 2015;98(6):913-922.

42. Veglia F, et al. Fatty acid transport protein 2 reprograms neutrophils in cancer. Nature. 2019;569(7754):73-78.
43. Chang CJ, et al. Targeting tumor-infiltrating Ly $6 \mathrm{G}^{+}$myeloid cells improves sorafenib efficacy in mouse orthotopic hepatocellular carcinoma. Int JCancer. 2018;142(9):1878-1889.

44. Granot Z. Neutrophils as a therapeutic target in cancer. Front Immunol. 2019;10:1710.

45. Ingram JP, et al. A nonpyroptotic IFN- $\gamma$-triggered cell death mechanism in nonphagocytic cells promotes salmonella clearance in vivo. J Immunol. 2018;200(10):3626-3634.

46. Dobin A, et al. STAR: ultrafast universal RNA-seq aligner. Bioinformatics. 2013;29(1):15-21.

47. Love MI, et al. Moderated estimation of fold change and dispersion for RNA-seq data with DESeq2. Genome Biol. 2014;15(12):550.

48. Subramanian A, et al. Gene set enrichment analysis: a knowledge-based approach for interpreting genome-wide expression profiles. Proc Natl Acad Sci U S A. 2005;102(43):15545-15550.

49. Raudvere U, et al. g:Profiler: a web server for functional enrichment analysis and conversions of gene lists (2019 update). Nucleic Acids Res. 2019;47(W1):W191-W198. 